\title{
Digital Proximity Tracing in the COVID-19 Pandemic on Empirical Contact Networks
}

\section{Giulia Cencetti}

Fondazione Bruno Kessler

\section{Gabriele Santin}

Fondazione Bruno Kessler https://orcid.org/0000-0001-6959-1070

\section{Antonio Longa}

Fondazione Bruno Kessler https://orcid.org/0000-0003-0337-1838

\section{Emanuele Pigani}

Fondazione Bruno Kessler https://orcid.org/0000-0002-0566-0759

\section{Alain Barrat}

French National Centre for Scientific Research https://orcid.org/0000-0001-8683-269X

\section{Ciro Cattuto}

ISI Foundation

\section{Sune Lehmann}

Technical University of Denmark https://orcid.org/0000-0001-6099-2345

\section{Marcel Salathé}

Ecole Polytechnique Fédérale de Lausanne

\section{Bruno Lepri ( $\square$ lepri@fbk.eu )}

\author{
Fondazione Bruno Kessler
}

\section{Article}

Keywords: digital contact tracing, COVID-19, control outbreaks

Posted Date: July 21st, 2020

DOI: https://doi.org/10.21203/rs.3.rs-41017/v1

License: (c) (i) This work is licensed under a Creative Commons Attribution 4.0 International License. Read Full License

Version of Record: A version of this preprint was published at Nature Communications on March 12th, 2021. See the published version at https://doi.org/10.1038/s41467-021-21809-w. 


\title{
Digital Proximity Tracing in the COVID-19 Pandemic on Empirical Contact Networks
}

4 and B. Lepri ${ }^{1, \star}$

$5{ }^{1}$ Fondazione Bruno Kessler, Trento, Italy

$6 \quad{ }^{2}$ University of Trento, Trento, Italy

$7{ }^{3}$ Aix Marseille Univ, Université de Toulon, CNRS, CPT, Turing Center for Living Systems, Marseille, France

$8{ }^{4}$ Tokyo Tech World Research Hub Initiative (WRHI), Tokyo Institute of Technology, Tokyo, Japan

$9{ }^{5}$ University of Turin, Turin, Italy

$10{ }^{6}$ ISI Foundation, Turin, Italy

$1{ }^{7}$ Technical University of Denmark, Copenhagen, Denmark

2 École Polytechnique Fédérale de Lausanne (EPFL), Lausanne, Switzerland

$\dagger$ These authors contributed equally to this work.

^Corresponding author: lepri@fbk.eu

\begin{abstract}
Digital contact tracing is increasingly considered as a tool to control infectious disease outbreaks. As part of a broader test, trace, isolate, and quarantine strategy, digital contract tracing apps have been proposed to alleviate lock-downs, and to return societies to a more normal situation in the ongoing COVID-19 crisis ${ }^{112}$ Early work evaluating digital contact tracing ${ }^{1 / 3}$ did not consider important features and heterogeneities present in real-world contact patterns which impact epidemic dynamics ${ }^{45}$ Here, we fill this gap by considering a modeling framework informed by empirical high-resolution contact data to analyze the impact of digital contact tracing apps in the COVID19 pandemic. We investigate how well contact tracing apps, coupled with the quarantine of identified contacts, can mitigate the spread of COVID-19 in realistic scenarios such as a university campus, a workplace, or a high school. We find that restrictive policies are more effective in confining the epidemics but come at the cost of quarantining a large part of the population. It is possible to avoid this effect by considering less strict policies, which only consider contacts with longer exposure and at shorter distance to be at risk. Our results also show that isolation and tracing can help keep re-emerging outbreaks under control provided that hygiene and social distancing measures limit the reproductive number to 1.5. Moreover, we confirm that a high level of app adoption is crucial to make digital contact tracing an effective measure. Our results may inform app-based contact tracing efforts currently being implemented across several countries worldwide
\end{abstract}

\section{Introduction}

As of mid-June 2020, the COVID-19 pandemic has resulted in over 7.5 millions detected cases worldwide, $\frac{12}{12}$ overwhelming the healthcare capacities of many countries and thus presenting extraordinary challenges for governments and societies ${ }^{13-16}$ At present, no effective pharmaceutical treatments are known, and a vaccine is estimated to be approximately one year away ${ }^{[17}$ Nonpharmaceutical interventions (i.e. social distancing, wearing masks and reinforced hygiene) are 
therefore currently the main path towards mitigating the intensity of the pandemic and returning society to near-normal functioning without substantial new outbreaks.

Rigorous restrictions such as lock-downs and quarantine have prove effective in many countries as a measure to curb the spread of SARS-CoV-2, limit contagions and reduce the effective reproduction number $R_{e} e_{13 / 18-25}$ Many areas have now entered a transition phase, slowly lifting the restrictions. This transition phase is fragile, and an effective and affordable long-term plan is required to avoid resurgences of infections and new outbreaks. ${ }^{26}$ This is particularly pressing given the possibility that the COVID-19 pandemic will come in waves as anticipated by several early models, ${ }^{27 \mid 28}$ and since the fraction of the population which has been infected is still far too low to provide herd immunity. ${ }^{16}$

Despite their efficacy, large-scale quarantine and lock-down strategies carry enormous costs. ${ }^{19}$ Moreover, population-wide measures are non-specific: in a situation where most of the population is not infected, population-wide lock-downs are far from optimal, and interventions at smaller scale, selectively targeting individuals at higher risk of spreading the disease, are more desirable.

While the testing and isolation of symptomatic cases is certainly crucial, it is insufficient in the case of SARS-CoV-2, since there is clear evidence of presymptomatic transmission, ${ }^{1129130}$ and a fraction of infected individuals do not develop symptoms at all. ${ }^{31 / 32}$ Thus, the identification and isolation of infected cases must be coupled with a strategy for tracing their contacts who may have become infected. That way, contacts who may pass on the infection before the onset of symptoms, can then be quarantined and their health status monitored ${ }^{33}$ In this context, recent modeling studies have shown $\sqrt{1134-36}$ that contact tracing may reduce epidemic spreading, and that the efficacy of its realization - contact identification and timing - plays a pivotal role for mitigation.

Traditional contact tracing is performed manually, i.e. by interviews of cases. While this ensures a thorough assessment of the actual risk of individuals who have been in contact with these cases, it is slow and labor intensive ${ }^{37+39}$ and can be efficiently implemented only when the number of infected individuals is low. In addition, the accuracy of manual tracing is limited by the ability to recall and identify close proximity contacts: contacts without a social connection have a lower probability of being recalled, and contact duration is in general overestimated in retrospective surveys. ${ }^{40 \mid 41}$ Thus, technologies based on proximity sensors are currently being considered to complement manual tracing. Specifically, the idea is to leverage the widespread dissemination of smartphones to develop proximity-sensing apps based on the exchange of Bluetooth signals between smartphones, ${ }^{1 / 6-11 / 42[43}$ which makes it possible to build privacy-preserving contact tracing frameworks ${ }^{6}$ The efficacy of app-based contact tracing has been discussed in several recent papers. ${ }^{2 / 44}[47]$ Here, we start from the work by Fraser et al. $\frac{\sqrt{3}}{3}$ recently adapted to the case of COVID-19.1] This work 
describes the evolution of the spread using recursive equations describing the number of infected individuals in a homogeneously mixed population, taking into account the (evolving) infectiousness of the infected individuals. These equations are modified by two effective parameters, $\varepsilon_{I}$ and $\varepsilon_{T}$, to represent the ability to identify and isolate infected people, and to correctly trace their contacts, respectively. Assuming an exponential growth for the number of infected people (applicable in early phases of an epidemic outbreak) the authors study how the growth rate depends on the intervention parameters. This approach, however, has several limitations, both from the point of view of modeling and visa-vis applicability. First, the assumption of full homogeneous mixing is an important limitation in epidemic modeling, $\frac{454849}{40}$ and realistic social network architectures might be particularly relevant for contact tracing. ${ }^{44}$ Second, the mathematical framework is limited to exponential growth. Third, the parameters $\varepsilon_{I}$ and $\varepsilon_{T}$ are assumed to be independent.

Here, to better understand the concrete efficacy of real-world contact tracing, we expand this approach with respect to these three aspects.

First, we provide a realistic quantification of the tracing ability by performing simulations of spreading processes and of contact tracing strategies on real-world data sets collected across different social settings (i.e., a university campus, a workplace, a high school). $50-52$ This allows us to estimate the actual "tracing ability" parameter $\varepsilon_{T}$ for different possible tracing policies (i.e., the thresholds considered to define a contact measured by the app as "at risk") and for different values of $\varepsilon_{I}$ (Section 4.1). The parameter $\varepsilon_{T}$ can then be inserted into the mathematical model to study the impact of the tracing policy on the spread. By making use of real contact network data sets, we capture complex interaction structures that are necessary for a realistic quantification of this parameter, although the data sets represent small groups compared to e.g. a city or a country. It is important to emphasize that the evolution of epidemic is based on a solid theoretical model and using real data of social contacts does not affect the reliability of results and does not limit them to special settings, as the contact data are only used to simulate the impact of isolation and tracing in different contexts and under different restrictions. In order for app-based contact tracing to work properly, it is necessary that the potential infection events estimated by smartphones constitute a reasonable proxy for real-world infection dynamics. However, little is currently known about the relationship between actual biological infections and the type of proximity interactions detected using smartphones. Here, we assume that the probability of a contagion event occurring during a proximity event between a healthy and an infected individual depends both on the duration and on the distance between those individuals ${ }^{53 / 54}$ (along with other epidemiological variables such as the infectiousness of the individual - see Supplementary Information A). Note that, as Bluetooth signal strength is not trivially converted to a distance,$[55]$ we rely - as the apps being currently developed will $d c^{6}-$ on the received signal strength as a proxy for distance. 
Secondly, we restructure and generalize the mathematical framework of the approach proposed by Fraser.13 This allows us to completely avoid assumptions regarding the functional form of the epidemic growth, making the setting applicable to any possible evolution shape and any phase of the epidemic. Moreover, we have modified the epidemiological aspect of the model according to recent literature on COVID-19, $\sqrt[56-58]{5}$ to fully consider asymptomatic cases and the delay in isolating individuals after they are identified as infected (Supplementary Information B).

Our third key contribution concerns the modeling and the detailed investigation of the contact tracing procedure. We devote a particular attention to the tracing parameter $\varepsilon_{T}$, which unavoidably depends on the ability to detect infected people whose contacts can then be traced: in other words, $\varepsilon_{T}$ is not independent from $\varepsilon_{I}$. Moreover, when gathering information on the recent contacts of an infected individual, it is obviously not possible to know which interactions, if any, really did correspond to a contagion event. Any contact tracing policy is thus based on thresholds on the duration and proximity of a contact to define the associated infection risk. Among 'risky' contacts, some correspond to infections while others do not. The latter correspond to "false positives", i.e., non-infected individuals who will be quarantined. Similarly, among the contacts considered as nonrisky by the contact tracing, some might actually be infected ("false negatives"). The use of realworld data makes it possible to evaluate the number of false positives and negatives for various policies, together with their effectiveness in containing the spread. These outcomes represent crucial information as they might determine the usefulness of contact tracing apps. On the one hand, a low number of quarantined, or mis-calibrated policies, can unwittingly omit many potential spreaders. On the other hand, highly restrictive policies might require to quarantine large numbers of individuals, including non-infected people, with a consequent high social cost.

Overall, our approach allows us to evaluate the effect of different contact tracing policies, not only on the disease spread but also in terms of their impact on the fraction of quarantined individuals. We develop our main analysis adopting the most recently described epidemiological characteristics of COVID-19, and we consider a scenario with a reproduction number $R_{0}=1.5$, representative of a situation of a re-emerging outbreak that may be faced after the release of the lock-down measures. Moreover, we investigate a range of possible values of $R_{0}$ and, by clearly identifying the relevant variables, we can provide insights on how to tune and adapt policies to be maximally effective. This novel combination of a well-established epidemic model with state-of-the-art, empirical interaction data collected via Bluetooth technologies or similar radio-based proximity-sensing methods, allows us to understand the role played by intrinsic limitations of app-based tracing efforts, affording an unprecedented viewpoint on the ambition of achieving containment with app-based interventions. Namely, we are able to test and quantify the role that a real contact network plays both for the infectiousness of a contact and for the ability of a policy to detect it and to respond optimally. 
Identifying a tracing policy that is able to contain the epidemic is a non-trivial task, and our aim is to quantify and describe the properties that makes a policy effective, in terms of duration and signal strength thresholds, tracing period of time and isolation efficiency. Furthermore, we show that isolation and tracing measures are only as effective as the technology they rely upon: No tracing is possible if adequate proximity and time resolutions are not available.

Even if it is clear that the choice of a particular policy should be primarily guided by its effectiveness in containing the virus, we find that not all successful policies are equal. In particular, we demonstrate that beyond a certain accuracy, stricter policies do not improve the containment. Hence, comparing policies also at this level allows one to improve their design and to reduce their side effects.

\section{Results and Discussion}

We evaluate the effect of measures based on the deployment of a digital contact tracing app on the mitigation of the Covid-19 pandemic. As we do not consider geography nor large-scale mobility, our modeling can be considered as referring to a limited geographical area, similarly to previous modeling efforts! $[135$

In particular, we assume two types of interventions to limit the spread of the virus. First, infected individuals are isolated when they are either symptomatic and self-reporting or if they are identified through randomized testing. Second, individuals who have had a potentially contagious contact with identified infected individuals can be preventively quarantined, following an exposure notification via an app on their smartphone. Schematically, if a detected infected individual has the app, the anonymous keys that her/his device has been broadcasting through Bluetooth in the past few days are exposed. The app of the individuals with whom $\mathrm{s} / \mathrm{h}$ has been in contact in the past days recognizes these keys as stored on their own device and calculates a risk score. If the risk score obtained by an individual is above a certain threshold (determined by the considered policy), the contact is "at risk" and the individual is assumed to go into quarantine. We refer to Troncoso et al ${ }^{6}$ for more details on the implementation on privacy preserving proximity tracing. Our research question is whether or not it is possible to contain a COVID-19 outbreak by means of such measures.

We introduce the concept of potentially contagious contact into a mathematical framework where the epidemic evolution is governed by a model based on recursive equations, inspired by the work of Fraser et al.,$^{3}$ and recently adapted to the Covid-19 case..$^{1}$ This model quantifies the number of newly infected people at each time interval, given a characterization of the disease in terms 

quantifies instead the efficacy of contact tracing).
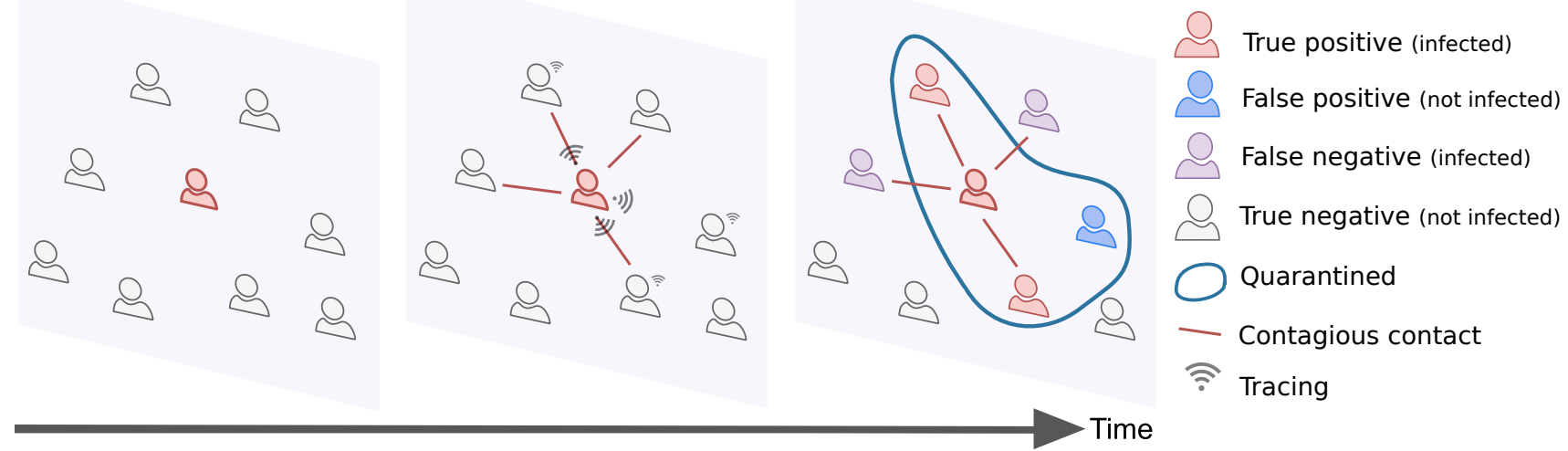

of infectiousness and manifestation of symptoms. the two interventions described above, whose effectiveness are quantified by two parameters $\varepsilon_{I}, \varepsilon_{T}$ varying from 0 to 1 (where $\varepsilon_{I}=0$ means "no isolation" and $\varepsilon_{I}=1$ represents a perfectly successful isolation of all individuals who are found to be infected, either via self-reporting or testing; $\varepsilon_{T}$

Here, particular attention is devoted to the study of the dependence of $\varepsilon_{T}$ on $\varepsilon_{I}$, and to assess which policies are achievable given the present technology and resources. To this aim, we couple this model with a realistic quantification of the effect of these two measures based on real-world contact and interaction data. The following results are indeed obtained by simulations using the Copenhagen Networks Study (CNS) datase $\left[\frac{50}{5}\right.$ that describes real proximity relations of smartphone users measured via Bluetooth (see Section 4.1). Moreover, we present in the Supplementary Information simulations performed using two other datasets collected by the SocioPatterns collaboration with a different type of wearable sensors! $51 / 52$

We emphasize that in each case, a realistic quantification of the tracing ability is obtained by simulating the epidemics on a dataset, but the controllability of the disease is assessed by the general mathematical framework and is therefore not bounded by specific datasets.

Figure 1: The contacts among users of the contact tracing app are registered through via the app. As soon as an individual is identified as infected $\mathrm{s} /$ he is isolated, and the tracing and quarantine policy is implemented. Depending on the policy design, the number of false positives and false negatives may vary significantly.

We consider five different policies (Table 1) that correspond to different threshold levels on the signal intensity, considered as a proxy of distance, and on the duration of the contact. Recall that Bluetooth does not measure distances per se, therefore all real-world implementations are based on thresholds on Received Signal Strength Indicator (RSSI) values. We additionally assume that each individual app stores the anonymous IDs received from other apps, representing a history of 
the past contacts over the past $n$ days. Here, we consider $n=7$ days, as we found (Supplementary Information C.1] that a longer memory does not produce any significant improvement. Overall, this implies that we consider a simplified version of the app, which does not compute risk scores but is simply able to remember the contacts corresponding to a sufficiently close and long-lasting proximity during $n$ days, while contacts below the thresholds are not stored. In addition, each policy is tested with the isolation efficiency values $\varepsilon_{I}=0.2,0.5,0.8,1$, which encode isolation capacities ranging from rather poor to perfect isolation of any symptomatic or tested positive person.

\begin{tabular}{|c|c|c|c|}
\hline ID & $\begin{array}{c}\text { Signal strength } \\
(\mathrm{dBm})\end{array}$ & $\begin{array}{c}\text { Duration } \\
(\mathrm{min})\end{array}$ & $\begin{array}{c}\text { Contact } \\
\text { percentage }\end{array}$ \\
\hline - Policy 1 & -73 & 30 & $2.2 \%$ \\
\hline - Policy 2 & -80 & 20 & $7.3 \%$ \\
\hline - Policy 3 & -83 & 15 & $13.4 \%$ \\
\hline - Policy 4 & -87 & 10 & $25.9 \%$ \\
\hline - Policy 5 & -91 & 5 & $56.7 \%$ \\
\hline
\end{tabular}

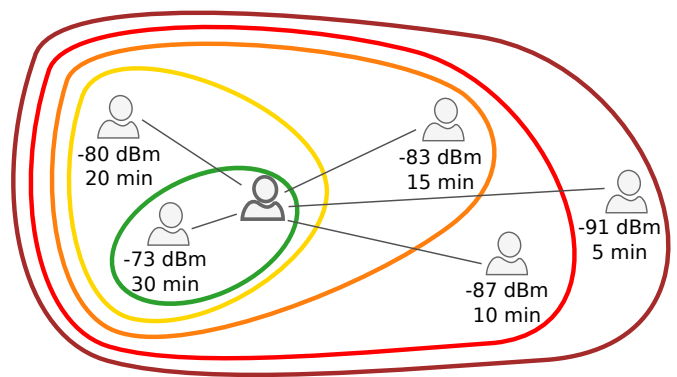

Table 1: Parameters defining the policies, and fraction of the total number of interactions of the CNS dataset that they are able to detect. A larger value of the magnitude of the signal strength tends to correspond to a larger distance, such that in the second column the thresholds go from the least to the most restrictive policy.

A fundamental difference between the policies we consider is related to the fraction of contacts that are stored by the app. Figure 3 shows the distributions of RSSI and contact durations of for the interactions in the CNS dataset. Most contacts have short duration and low signal strength (and are thus likely random contacts), but long lasting durations are also observed, with overall a broad distribution of contact durations as typical from data on human interactions. ${ }^{55[59}$ The thresholds defined by the tracing policies determine the fraction of these contacts that are traced by the app: only the contacts within the specified regions are considered when determining who is alerted by the app and hence quarantined. Even slight variations in the tracing policy thresholds may strongly influence the capacity to identify the contacts corresponding to the highest risks of infection, as shown in Figure 3 by comparing the RSSI and contact duration distributions with the infectiousness curves (as functions of the signal strength and of the contact duration between individuals, see Supplementary Information A).

Finally, we consider two additional policies (Table 7) in Supplementary Information C.5: those policies use either close range but short exposure interactions or long range but long exposure interactions. 


\subsection{Isolation and tracing depend strongly on the fraction of asymptomatic cases}

We first obtain an overview of how the control parameters impact the spread of the disease in an idealized model where $\varepsilon_{I}, \varepsilon_{T}$ can take arbitrary values. In this way we can explore the full range of $\varepsilon_{I}$ and $\varepsilon_{T}$ and understand their effect - as in Ferretti et al ${ }^{11}$ Later we derive the sets of feasible configurations that can occur in reality based on simulations in contact networks. Here and in the following, we assume that the reproduction number is $R_{0}=1.5$. In addition, we consider values of $R_{0}=1.2$ and $R_{0}=2$ as more optimistic and more pessimistic scenarios, respectively (Supplementary Material $A$ ).

For each value of $\varepsilon_{I}, \varepsilon_{T}$, we use the model to predict the evolution of the number of newly infected people $\lambda(t)$ at time $t$ up to a time $T=50$ days, and we report the average growth or decline in the last 10 days. All numerical solutions of the continuous model reach a stationary growth or decline regime (constant growth or decline rate of $\lambda(t)$ ). A negative number indicates that the epidemic is declining, while a positive one corresponds to growth (uncontained epidemics).

An important ingredient of the model is given by the probability $s(\tau)$ for an infected individual to be recognized as infected within a period of time $\tau$, either via testing after the symptoms onset, or via randomized testing ${ }^{25}$ The ideal case in which all infected people can eventually be identified $(s(\tau)$ approaching 1 for large times) is reported in Figure 2a: this represents the best case scenario. We remark that this is the setting considered in the previous studies of this model $!^{113} \mathrm{Next}$, we assume instead that $40 \%$ of infected individuals are asymptomatic ${ }^{1 / 31 / 32 / 60 \mid 61}$ and that only symptomatic individuals are identified: no randomized testing is performed. We represent asymptomatics by considering that the probability of an infected individual to display symptoms is a growing function of time that never reaches the value 1 . In this case, the model predicts epidemic containment for the upper half of the values of the parameters $\varepsilon_{I}$ and $\varepsilon_{T}$ (Figure 2C).

In the following, we assume an alternative scenario where instead the asymptomatics (whose distribution of identification times follows the definition of $s(\tau)$ in Supplementary Information A account for the $20 \%$ of the infected population ${ }^{6263}$ Indeed, there is still no agreement in the scientific community about the fraction of asymptomatic infections for Covid-19; therefore different possible scenarios should be considered.25 We remark (see Supplementary Information A) that this scenario is equivalent to assuming that the symptomatics instead represent $60 \%$ of the infected population, and that $50 \%$ of asymptomatic infected are identified by a policy of randomized testing ${ }^{25}$ in addition to the symptomatic individuals. This is our baseline for the following investigations and the resulting model predictions are plotted in Figure 2b. Note that we moreover take into account in all settings a delay of 2 days between the detection of an infected individual and the 
time this information becomes available to the health authorities.

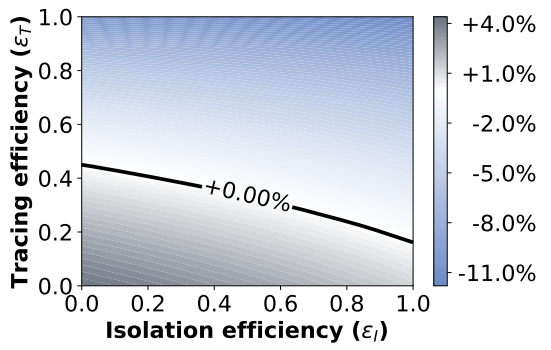

(a)

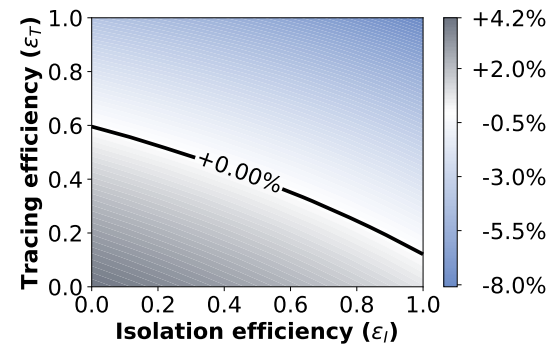

(b)

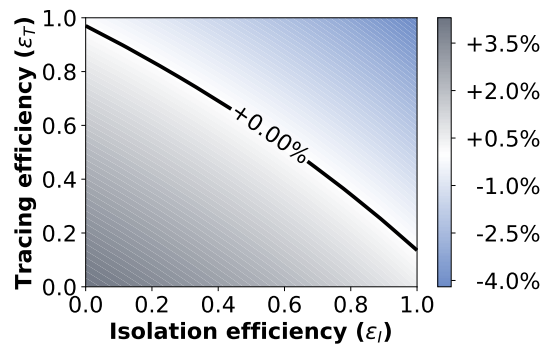

(c)

Figure 2: Growth or decrease rate of the number of newly infected individuals, assuming either that all the infected people can eventually be identified and isolated (Figure 2a); or that only symptomatic people can be isolated with $20 \%$ of infected individuals asymptomatic (Figure 2b); or that only symptomatic people can be isolated with $40 \%$ of infected individuals asymptomatic (Figure 2c). In all settings the cases are reported with a delay of 2 days.

\subsection{Real data restrict the range of successful policies}

We have run numerical simulations of the disease spread on the CNS dataset, implementing the different policies to determine their impact in mitigating the epidemic.

Figure 3 (bottom right panel) provides an illustration of the insights obtained by these simulations based on real contact data sets, by showing the distribution of the time elapsed between an infection event and the successive contacts of the infected individual for Policy 5 (see the table in Figure 4) and for $\varepsilon_{I}=0.8$. Most contacts occur before infected individuals reach their maximum infectiousness, but a non-negligible number of contacts occur while the individual is highly infectious.

By considering the five different policies of Table 1 in terms of which contacts are considered at risk and thus kept by the app, and running corresponding simulations for $\varepsilon_{I}=0.2,0.5,0.8,1$, we obtain in each case the actual value of $\varepsilon_{T}$ which quantifies the quality of the tracing policy (see Section 4.1. This value therefore ceases to be an arbitrary parameter: it is a direct consequence of the policy, the value of $\varepsilon_{I}$ and the contact data. We then plug the values $\left(\varepsilon_{I}, \varepsilon_{T}\right)$ into the idealized model, observe in which region of the diagrams of Figure 2 they fall, and deduce whether containment is achieved or not by this policy and this value of $\varepsilon_{I}$.

The results, reported in Figure 4 (center right), reveal that not all parameter configurations are feasible. In particular, the largest value of the tracing efficacy $\varepsilon_{T}$ can be reached only for Policies 4 and 5 . Policies 1, 2 and 3 still manage to reach the epidemic containment phase if $\varepsilon_{I}$ is large 

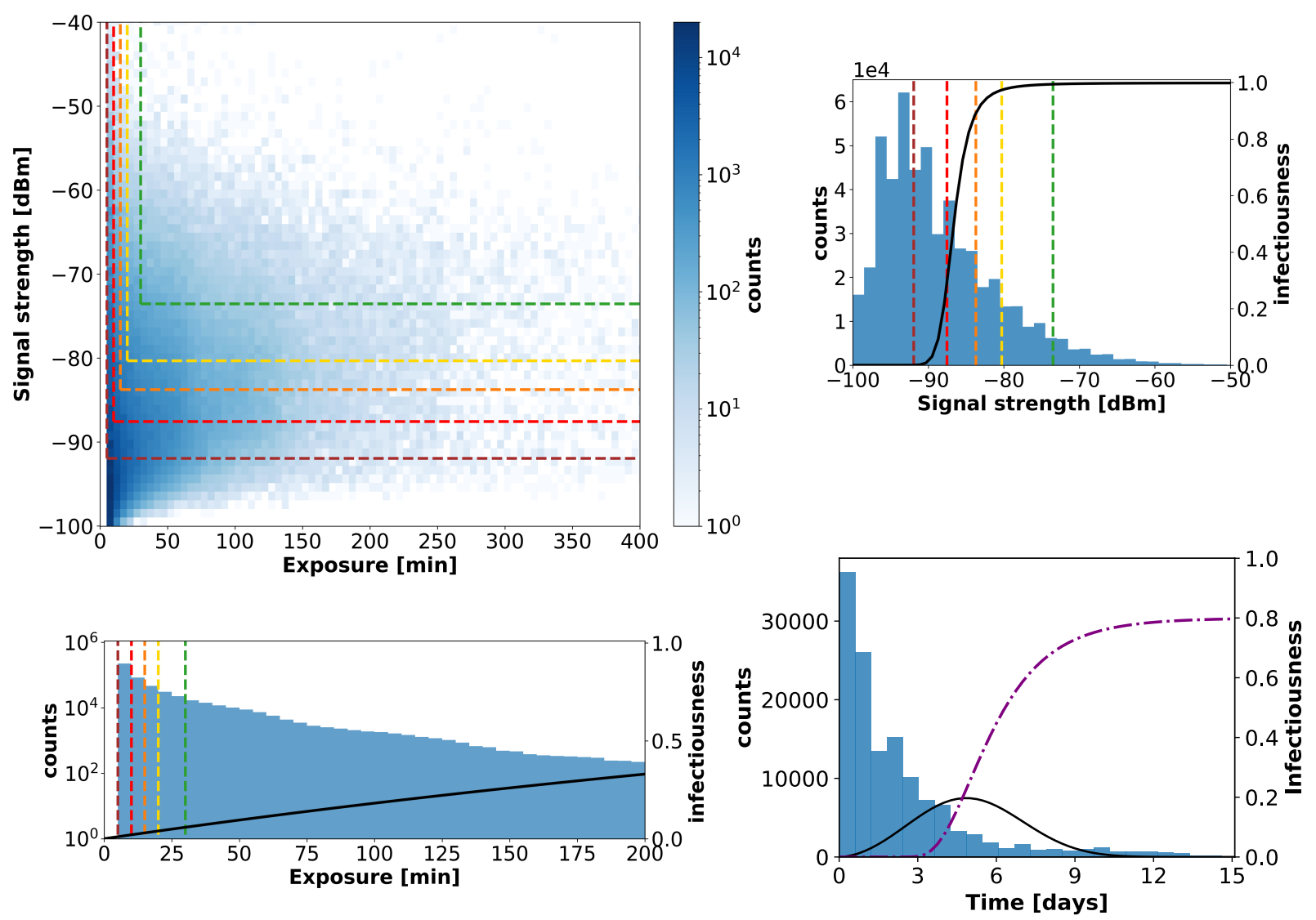

Figure 3: The top left panel shows a scatterplot of signal strength vs duration for all contact events in the CNS dataset, and displays the thresholds defining the various policies: the contacts identified as "at risk" are those included in the areas identified by the colored lines. Top right and bottom left panels separately depict the distributions of signal strength and duration, together with the infectiousness functions $\omega_{\text {dist }}$ and $\omega_{\text {exposure }}$ respectively (black curves), see Table 3 in Supplementary Information for their analytical form. The bottom right panel shows the distribution of time elapsed between the infection of an individual and their successive contacts, obtained with $\varepsilon_{I}=0.8$ and for Policy 5 in the CNS dataset. The black curve shows the normalized infectiousness $\omega(\tau)$ as a function of time, and the purple dashed line is the cumulative probability to detect an infected person $s(\tau)$.

enough, and only Policy 1 is too restrictive in its definition of risky contacts, and thus ineffective, for $\varepsilon_{I}=0.5$. Furthermore, in all cases an isolation efficacy of only $\varepsilon_{I}=0.2$ is not sufficient, no matter which policy is used. We also note that Policies 4 and 5 do not differ in their results, although the number of contacts retained are quite different (as seen in Figure 3, top left): once a certain 

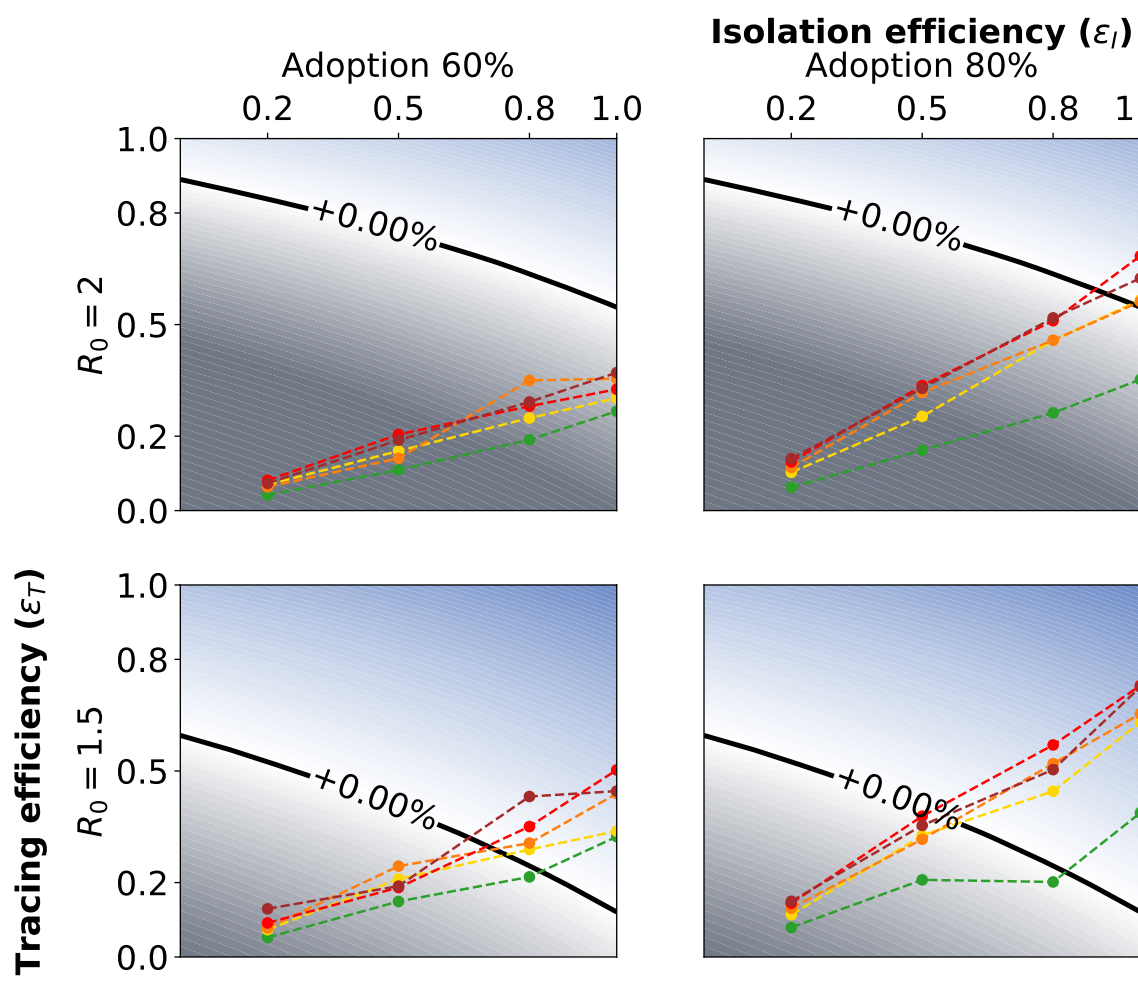
0.2
0.5
$0.8 \quad 1.0$

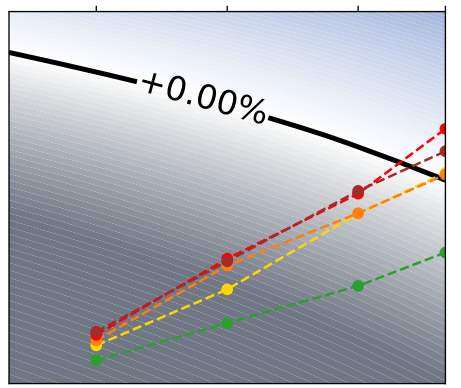

Adoption $100 \%$
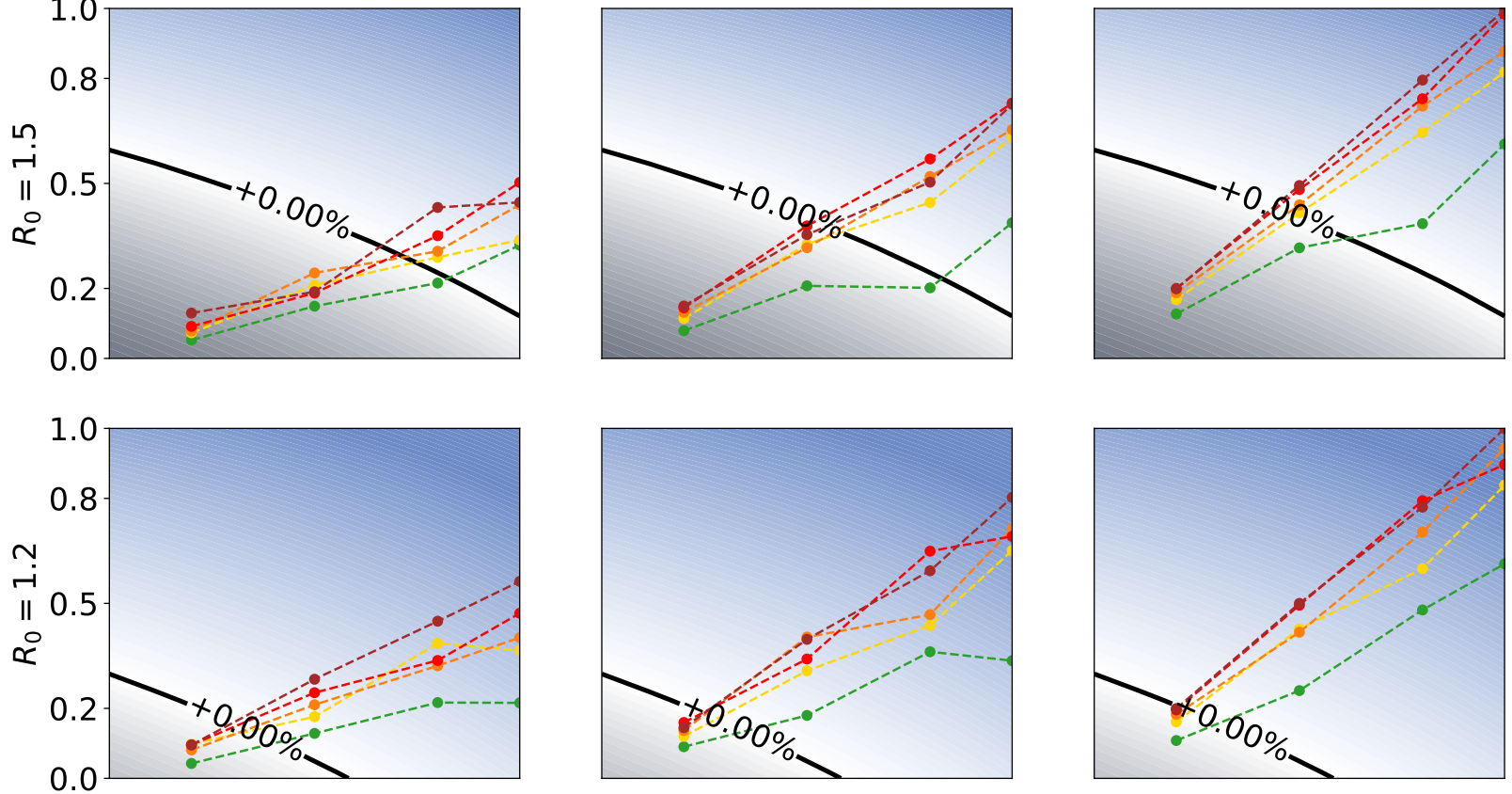

Figure 4: Tracing policy efficiency. Growth or decrease rate of the number of newly infected people assuming that symptomatic people can be isolated and that an additional $50 \%$ of asymptomatic can be identified via randomized testing. The points correspond to the parameter pairs such that $\varepsilon_{I}$ is an input and $\varepsilon_{T}$ an output of the simulations on real contact data, for the five policies. The different scenarios are defined by an app adoption level of $60 \%, 80 \%$, or $100 \%$ (from left to right), and by a value of $R_{0}$ equal to $2,1.5$, or 1.2 (from top to bottom). 


\subsection{Digital tracing enable containment in the face of larger reproduction numbers}

Until now, we have assumed that the only limit to reach a perfect contact tracing resides in the technology specifications of each policy. This implies however that the totality of the population adopts the app, something which is clearly unrealistic in practice. We thus repeated our simulations assuming that only a fraction of the population uses the app, while the remaining individuals are outside the reach of the tracing and quarantining policies, but they are still isolated whenever detected because symptomatic or through random testing (see Section 4.1.5).

We found that reducing the app adoption implies an important reduction in the tracing policies effectiveness. The first two columns of panels in Figure 4 report the results for an adoption of $60 \%$ and $80 \%$ respectively. If $R_{0}=2$, practically none of the policies is able to stop the spreading. However, this pessimistic scenario changes under the current working hypothesis of $R_{0}=1.5$ (second line of panels in Figure 4). An app adoption of $80 \%$ or even $60 \%$ is then sufficient to obtain good results: all policies except for Policy 1 manage to contain the spread for $\varepsilon_{I}=0.8$, and all of them for $\varepsilon_{I}=1$ (Figure 4, center row). The situation is even better with a smaller value of $R_{0}=1.2$. In this case, even in the case of an app adoption of only $60 \%$, all policies are effective as soon as the isolation efficacy is at least 0.5 (bottom left panel in Figure 4).

We observe that the tracing efficiency, which clearly varies considerably with different levels of app adoption, practically does not depend on $R_{0}$. Indeed, $\varepsilon_{T}$ only accounts for the fraction of secondary infections that are correctly traced, independently on the spread of the virus and the amount of infected people in the population.

We also note that the effect of a limited app adoption on the tracing efficiency $\varepsilon_{T}$ appears to be quadratic: a $60 \%$ app adoption reduces the efficiency roughly to its $40 \%$, while an $80 \%$ adoption reduces it to the $70 \%$ (see Supplementary Information C.3). This effect is explained by the fact that in order to trace a contact between two individuals it is necessary that both have the app installed.

These different scenarios highlight the efficacy of digital contact tracing and its fundamental role as a component of a larger effort including additional containment measures. Indeed, while in the absence of contact tracing a value of $R_{0}$ larger than one may rapidly lead to a new exponential outbreak that demands (possibly local) lockdown measures to be enforced, we clearly demonstrated that a digital tracing app, if sufficiently adopted, may stop the spread of the virus even with reproduction numbers up to $R_{0}=2$, depending on the isolation efficiency. 
Behind the scenes of the aggregated results of the previous section, there is a complex dynamic that merits further investigation. Indeed, the different tracing efficiencies are the macroscopic result of a different set of contacts considered as at risk. In some cases this produces the desirable effect of containing the spread, but side effects emerge as well. Indeed, some of the "at risk" contacts do not actually lead to a contagion event, while contacts classified as non risky might, since the spreading process is inherently stochastic. It is thus important to quantify the ability of each policy to discriminate between contacts on which the disease spreads and the others, in terms of false positives (quarantined individuals who were not infected) and false negatives (nonquarantined infected individuals). To visualize this behavior, we focus on the setting with $R_{0}=1.5$ and $\varepsilon_{I}=0.8$, with an app adoption of $80 \%$, since it is representative of a situation in which some policies are effective in containing the spread and others are ineffective (see Figure 4, center). The corresponding time evolution of the average number of false negatives and of false positives for each policy are shown in Figure 5 .

What matters in terms of virus containment is to rapidly reduce the number of infected people that have contacts and spread the virus. In the case of Policy 1, the level of false negatives remains quite high for the entire time, and it is never reduced to zero. For all other policies instead the curves of false negatives all reach similar levels. The curve drops to zero rapidly however only for the stricter policies, while for Policy 2 it remains at a higher level, showing that the spread is not contained early in the process.

The smaller number of false negatives for the effective policies comes however at the cost of an increased number of false positives, as shown in Figure $5 \mathrm{~b}$. In other words, as a policy becomes more effective in tracing actually infected people, it also leads to the quarantine of individuals that have not been infected but that had a contact classified as risky by the tracing policy. This is also made clear by the attack rate values reported in the table in Figure 5, corresponding to the fraction of true positives: starting from Policy 2 to Policy 5 , the number of quarantined people increases and the fraction of quarantined who are actually infected decreases. In all cases, the maximal number of false positives is very sensitive to the specific policy, contrarily to the number of false negatives. In particular, it appears from the analysis of Section 2.3 that Policies 3, 4 and 5 have a similar effectiveness to contain the epidemic and Figure 5 shows that they yield indeed similar numbers of false negatives, but their undesired side costs are different, as the broader definition of risky contacts of Policy 5 produces a larger number of false positives. This highlights once more the importance of fine-tuning of the chosen policy. 


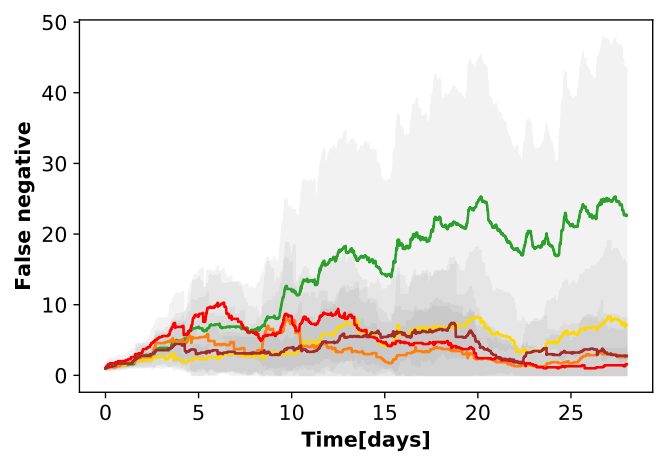

(a)

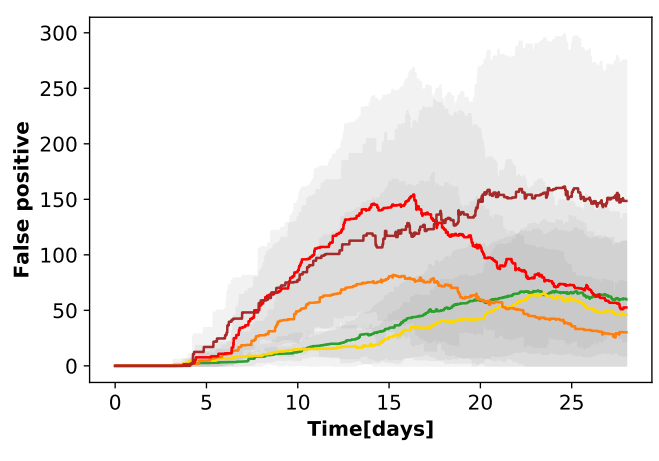

(b)

\begin{tabular}{|c|c|c|}
\hline & $\begin{array}{c}\text { Total } \\
\text { quarantines }\end{array}$ & $\begin{array}{c}\text { Attack } \\
\text { rate }\end{array}$ \\
\hline$\bullet$ & $145 \pm 102$ & $0.16 \pm 0.11$ \\
\hline$\bullet$ & $165 \pm 111$ & $0.097 \pm 0.04$ \\
\hline$\bullet$ & $203 \pm 145$ & $0.088 \pm 0.04$ \\
\hline$\bullet$ & $274 \pm 110$ & $0.061 \pm 0.02$ \\
\hline$\bullet$ & $282 \pm 159$ & $0.036 \pm 0.01$ \\
\hline
\end{tabular}

$282 \pm 159$

Figure 5: False positive and negative in quarantines with $80 \%$ app adoption. Temporal evolution of the numbers of false negatives (Figure $5 \mathrm{a}$ ) and false positives (Figure 5b) for the five different policies, assuming an isolation efficiency of $\varepsilon_{I}=0.8$, an $80 \%$ app adoption level. The graphs depict the mean and standard deviation over 20 independent runs. The table reports mean and standard deviation of the total number of distinct individuals who have been quarantined over the whole simulation timeline and the percentage of those among them who were effectively infected (true positive), corresponding to the attack rate.

\section{Conclusions}

In this study, we have analyzed the ability of digital tracing policies to contain the spread of Covid19 outbreaks using real interaction datasets to estimate the key effective parameters and to shed light on the practical consequences of the implementation of various app policies.

We found that the set of parameters that allow containment of the spread is strongly influenced by the fraction of asymptomatic cases. By first assuming an ideal setting where any pair of parameters $\varepsilon_{I}, \varepsilon_{T}$ is possible, we showed (Figure 2) that the area of the phase space representing the setting where it is possible to control the epidemic is reduced when considering $20 \%$ or, worst case scenario, $40 \%$ of asymptomatic individuals in the population, i.e. infected people that we cannot isolate nor contact trace and who therefore continue spreading the virus to their contacts. We remark that this is in contrast with the scenario considered in Fraser et al $\frac{3}{3}$ and in Ferretti et al., 1 where the entire infected population is assumed to become symptomatic eventually.

We tested five policies to define risky contacts that should be traced (Table 1), with different restriction levels. When implemented on real contact data measured by Bluetooth, this approach allows us to estimate, for each value of $\varepsilon_{I}$, the actual value of $\varepsilon_{T}$ and thus to determine the efficacy of each policy (colored points in Figure 4). Using these implementations on real data restricts the available values of the control parameters. This added layer of realism reveals that only the most 
restrictive policies can lead to epidemic containment.

Moreover, even for these policies, tracing is effective only if the isolation is effective: Policy 2 requires a perfectly effective isolation $\left(\varepsilon_{I}=1\right)$, while an $80 \%$ isolation is sufficient for Policies 3 , 4, and 5 (Figure 4). In particular, better tracing policies may work with a less effective isolation strategy.

Our results highlight how isolation and tracing come at a price, and allow us to quantify this price using real data: the policies that are able to contain the pandemic have the drawback that healthy persons are unnecessarily quarantined (Figure 5). In other words, achieving a rapid containment and a low number of false negatives requires accepting a high number of false positives. This stresses the importance of a fine tuning of the tracing and isolation policies, in terms of the definition of what represents a risky contact, to contain the social cost of quarantines.

Finally, we have shown that an insufficient app adoption may render any digital tracing effort helpless on its own, if the reproductive number is too high. In view of these results, bridging the gap between a realistic app adoption and the larger tracing capability required to contain the disease appears crucial. In particular, digital tracing in itself may not be enough if not complemented by a traditional manual tracing policy and/or other measures. This goal can be reached only with a joint effort of policy makers and health authorities in organizing an effective manual tracing, and of individual citizens in adopting the app.

Our study comes with a number of limitations. First of all we are focusing on a single kind of intervention in order to fight the spread of the epidemics, which is just based on isolation and tracing. This is in order to isolate the effect of an app-based containment, avoiding additional spurious effects. In order to see the effect of isolation and tracing when implemented with other restrictive measures, we applied the same numerical machinery for a range of possible values of $R_{0}$, representing various levels of reduction of the spread. We did not consider in detail how this reduction could be obtained through specific containment measures such as mask wearing, social distancing, travel restrictions, school closures, and so on.

Moreover, we have considered data corresponding to a few limited social environments (a university campus, a high school and a workplace) and we cannot provide an overall general study that includes multiple and differentiated contexts and their mutual interplay. Our study is focused on the state of the art interaction datasets, which are nevertheless designed to capture a very limited social environment when compared e.g. with an entire city. This limitation is due to the current lack of larger datasets involving people belonging to different environments, which would represent the general interactions within the population of a city or a larger geographical area. In addition, the implemented policies have been necessarily tailored to this specific DTU dataset, depending 
on the available values of RSSI supported by the used smartphones. Those might therefore differ in actual implementations currently under development, probably relying on a more advanced technology than that of 2014 .

Finally, our study is limited by the state of the current knowledge of the contagion modalities of the SARS-CoV-2 virus, and in particular its dependence on physical distance among people and the duration of their contacts. The curve of infectiousness has been designed based on previous contagion studies and on reasonable assumptions (also considering a reduced transmissibility of asymptomatic people). Should new insights emerge in the way the virus spreads, these could be easily incorporated into our model.

\section{Data and Methods}

The mathematical model we use includes several parameters characterizing the epidemic, and we define them following the most recent literature. Although this is a mere literature survey step, it is nevertheless of fundamental importance. Indeed, as we will see in the following, different assumptions at this level may lead to different predictions. The main parameters are:

- $s(\tau)$, which is the probability for an infected individual to be detected as infected (and thus isolated) within a time $\tau$ after infection, either via self reporting after the symptoms onset, or via randomized testing;

- $\beta(\tau)$, which is the infectiousness of an infected individual at time $\tau$ after infection: this is defined as the probability that an individual who is infected since a period of time $\tau$ infects a new susceptible individual upon contact, $\omega(\tau)$, multiplied by the reproduction number, $R_{0}$. The curve $\omega(\tau)$ takes into account a reduced infectiousness of asymptomatic people and the environment effect too, as hypothesized by Ferretti et al.$^{11}$ An alternative shape of the curve is discussed in Section A.3 of Supplementary Information;

- $\omega_{\text {data }}(\tau, s, e)$, which is the analogous of $\beta$ when applied to a real dataset: it depends on the duration $e$ of a contact and on the strength $s$ of the interaction, quantified by the strength of the signal exchanged by the devices registering the interaction.

Details on these choices are discussed in Supplementary Information A.

The realistic values for the parameters $\varepsilon_{I}, \varepsilon_{T}$ and the interplay between the containment policies and the virus spread are estimated from real-world interaction datasets. Namely, we consider datasets describing networks of real contacts in a population, where interactions are determined by 
means of exchange of Bluetooth signals between smartphones. We develop a numerical simulation of the virus spread on this network, and in particular we implement a realistic notion of infection transmission upon contacts, which follows the parameters defined above. In this framework, we simulate isolation and tracing policies, where the tracing policy is implemented by defining sensors resolutions (e.g., a contact is detected if a minimal signal strength or a minimal contact duration are registered). It is also possible to model the rate of adoptions of the app, or the memory length of past traced contacts. The effect of these specifications on the actual effectiveness of the isolation and tracing policies are then obtained as output of the simulation: we obtain hence real-world estimations of the parameters $\varepsilon_{T}$, from the input value of $\varepsilon_{I}$ (that encodes e.g. the testing capacity, or the effectiveness of tests). The details of this simulation, the definition of the policies, and the strategy used to estimate the control parameters are described in more detail in Section 4.1 .

In order to incorporate these data-dependent and possibly time-varying control parameters into the predictions, we develop a modified analysis of the continuous model and its discretization. In particular, both Fraser et al.$^{[3}$ and Ferretti et al $!^{1}$ analyze the model prediction only in the limit of an infinite time horizon, and the first study ${ }^{3}$ furthermore assumes that the number of infected can only have an exponential behavior, either growing or declining. We instead consider the model at finite times and develop a new discretization of the continuous model that requires no assumptions on the functional form of the growth or decline of the contagion. This allows us to simulate more complex regimes and better quantify the effect of the control parameters, and in particular to align the model timescales with the simulation time, and thus to use the realistic parameters estimated from the simulations, including the implementation of time-dependent policies. This approach is presented in Supplementary Information B

The overall output of the model is the predicted number $\lambda(t)$ of newly infected individuals at time $t$, and we are interested to study policies that contain the epidemic, i.e., such that $\lambda(t) \rightarrow 0$ as $t$ grows.

\subsection{Policy evaluation from real datasets}

We describe here the network simulation that leads to the estimation of the parameter $\varepsilon_{T}$.

\subsubsection{Description of the datasets}

For the simulations we use the interaction data from the Copenhagen Networks Study, ${ }^{50}$ which describes the interactions of 706 students, as registered by the exchange of Bluetooth signals 
between smartphones, for a period of one month. From the complete dataset we extract the proximity measures in the form of Bluetooth signal strength.

\subsubsection{Definition of a contact and contagion probability}

At each time instant, and for each node in the graph which is currently infected, a probability distribution is used to decide whether or not the virus is spread to each of its contacts. This probability is the product of three components, i.e.,

$$
\omega_{\text {data }}\left(\tau, s_{s}, e\right):=\omega(\tau) \cdot \omega_{\text {exposure }}(e) \cdot \omega_{\text {dist }}\left(s_{s}\right)
$$

and it quantifies what contacts are relevant for the disease transmission. The three components are:

- $\omega(\tau)$, the probability for an infected individual to transmit the disease at time $\tau$, appearing in Figure 3 (bottom right panel) in the case of the dataset of the previous Section;

- $\omega_{\text {exposure }}(e)$, the probability for an infected individual to transmit the disease given the duration $e$ of a contact, appearing in Figure 3 (bottom left panel);

- $\omega_{\text {dist }}\left(s_{s}\right)$, the probability for an infected individual to transmit the disease given the signal strength $s_{s}$ of a contact, appearing in Figure 3 (top right panel).

We refer to Table 3 in Supplementary Information for the definition of each of these distributions.

\subsubsection{Description of the spreading algorithm}

We develop an individual-based model for the virus spreading. Starting from the real contacts dataset, we construct a weighted temporal network, in which nodes represent people and edges stand for temporal and distance-weighted connections between them. The dynamics performed on the network is described by the following algorithm.

We start at time $t=0$ with an initial number $Y_{i}$ of infected people, each one infected from a time $t=-\tau_{i}$, sampled from a uniform distribution $\mathcal{U}([0 ; 10])$. Thereafter, at each time step $t$ of size $\delta=300 s$ :

- Each $\tau_{i}$ value is incremented by $\delta$. 
- If an individual $i$ is neither isolated nor quarantined, $\mathrm{s} / \mathrm{he}$ can infect each of her/his neighbors $j$ of with a probability $\omega_{\text {data }}\left(\tau_{i}, s_{i, j}, e_{i, j}\right)$ (see previous Section).

- Newly infected people are assigned $\tau=0$ and a time to onset symptoms $t+t_{o}$, where $t_{o}$ is extracted from the distribution onset time $(\cdot)$ defined in Table 3.

- If an individual is recognized as infected (either as symptomatic or by testing) but still not isolated, we isolate him/her with probability $\varepsilon_{I}$ and we quarantine all her/his contacts according to a policy, that is, all her/his contacts above a spatio-temporal threshold (see the next Section for a precise description of this policy).

- If a quarantined individual becomes symptomatic, we quarantine all her/his previous contacts (i.e., before entering quarantine) according to the above-mentioned policy.

\subsubsection{Policy implementation and evaluation}

In a realistic scenario the isolation efficiency $\varepsilon_{I}$ or, in other words, the ability to identify and consequently isolate an infected individual, is set by the number of tests that are implemented and by their accuracy, features whose identification is out of the scope of this work. We mention that the adoption of an app might have a positive effect on this quantity if the possibility of self-reporting when symptoms appear is implemented in the device.

Our main goal is to characterize the efficiency of contact tracing, quantified by $\varepsilon_{T}$. This is far more than a simple parameter that freely varies between 0 and 1 . Its definition is indeed affected by multiple contributions, involving both the containment measures efficiency and the policy decisions. In a general setting we can easily identify two main dependencies: $(i)$ the fraction of primary infected individuals who are actually identified, isolated and whose latest contacts are investigated (in other words, $\varepsilon_{T}$ should be directly proportional to $\varepsilon_{I}$, approximately corresponding to the fraction of possibly secondary infections that a tracing policy can try to reconstruct); (ii) the real contact tracing, because once an infected individual is isolated the contact tracing will reach only part of her/his previous contacts, depending on the chosen policy. Indeed, if we choose a policy where all the contacts, even the most long range distance and irrelevant, of an infected individual are traced and quarantined, we would probably end up with a total lock-down of the entire population. There is therefore a social cost of this action that should be considered and possibly minimized. On the other side, when we decide to quarantine only people who had a large probability of having had a contagious contact, we are probably underestimating the infected people, leaving some of them outside of reach of the quarantine effort. The error that we introduce when we decide, for practical reasons, not to trace all the contacts of an infected individual represents an important contribution 
to the limitation of the tracing efficiency. This is quantified by $e_{T}(t)$, which takes values between 0 and 1 and is in general time-dependent.

The value of $e_{T}$ is estimated from the numerical simulations on the real temporal networks of contacts as follows. Once an individual is isolated we trace her/his contacts according to the chosen policy, then we count the fraction of people that $\mathrm{s} /$ he has actually infected who remained outside of the quarantine. By averaging on individuals and time we obtain $\left\langle e_{T}\right\rangle$. The obtained value thus encodes the contribution of the chosen policy, adoption rate, duration of the memory of contacts and potentially the warning of only the direct contacts or also of contacts of contacts.

The tracing efficiency can therefore be defined as the product of the two independent factors:

$$
\varepsilon_{T}=\varepsilon_{I}\left(1-\left\langle e_{T}\right\rangle\right)
$$

such that we obtain the maximum efficiency only if isolation is perfect and the quarantine error $\left\langle e_{T}\right\rangle$ is zero.

\subsubsection{Varying the app adoption levels}

When modeling different levels of adoption of the app we implement the following procedure: we extract at the beginning of each simulation a random list of users, that will act as non adopters. During the simulation these agents will contribute to the spread of the virus and will be subject to isolation whenever detected as infected, as any other individual, but in that case their contacts cannot be traced. Moreover they do never appear in any contact list, and thus they are never quarantined. In practice we simulate the fact that a contagious contact is recorded only if both the infectious and the infected have the app.

We make the simplifying assumption that the app influences only the quarantining of individuals, but not the isolation policy. Namely, we assume to be able to detect and thus isolate an infected individual independently of the app, while we are able to trace the contacts only between pairs of app adopters.

Author contributions: G.C, G.S., and B.L. conceived the idea. G.C., G.S., A.L., E.P performed the analytical calculations and numerical computations. All the authors contributed to research design, analytical development, critical revisions, and wrote the paper.

Declaration of competing interest: The authors declare that they have no known competing financial interests or personal relationships that could have appeared to influence the work reported in this paper. 
Acknowledgements: The authors would like to thank Esteban Moro, Alex Sandy Pentland, and Fabio Pianesi for early discussions and useful comments, Stefano Merler for the feedback on the design of the infectiousness parameters for COVID-19, and Valentina Marziano, Lorenzo Lucchini and Luisa Andreis for the discussion and general support. This study was partially supported by the ANR project DATAREDUX (ANR-19-CE46-0008-01) to AB.

\section{References}

[1] Luca Ferretti, Chris Wymant, Michelle Kendall, Lele Zhao, Anel Nurtay, Lucie Abeler-Dörner, Michael Parker, David Bonsall, and Christophe Fraser. Quantifying SARS-CoV-2 transmission suggests epidemic control with digital contact tracing. Science, 2020.

[2] Christophe Fraser, Lucie Abeler-Dörner, Luca Ferretti, Michael Parker, Michelle Kendall, and David Bonsall. Digital contact tracing: comparing the capabilities of centralised and decentralised data architectures to effectively suppress the COVID-19 epidemic whilst maximising freedom of movement and maintaining privacy. https://github.com/BDI-pathogens/ covid-19_instant_tracing, 2020.

[3] Christophe Fraser, Steven Riley, Roy M. Anderson, and Neil M. Ferguson. Factors that make an infectious disease outbreak controllable. Proceedings of the National Academy of Sciences, 101(16):6146-6151, 2004.

[4] R. Pastor-Satorras and A. Vespignani. Epidemic spreading in scale-free networks. Phys. Rev. Lett., 86:3200-3203, 2001.

[5] Alain Barrat, Marc Barthelemy, and Alessandro Vespignani. Dynamical processes on complex networks. Cambridge university press, 2008.

[6] Carmela Troncoso, Mathias Payer, Jean-Pierre Hubaux, Marcel Salathé, James Larus, Edouard Bugnion, Wouter Lueks, Theresa Stadler, Apostolos Pyrgelis, Daniele Antonioli, Ludovic Barman, Sylvain Chatel, Kenneth Paterson, Srdjan Čapkun, David Basin, Jan Beutel, Dennis Jackson, Marc Roeschlin, Patrick Leu, Bart Preneel, Nigel Smart, Aysajan Abidin, Seda Gürses, Michael Veale, Cas Cremers, Michael Backes, Nils Ole Tippenhauer, Reuben Binns, Ciro Cattuto, Alain Barrat, Dario Fiore, Manuel Barbosa, Rui Oliveira, and José Pereira. Decentralized privacy-preserving proximity tracing, 2020.

[7] Alexis Dudden and Andrew Marks. South Korea took rapid, intrusive measures against Covid19 - and they worked. The Guardian, 20, 2020. 
[8] Nuria Oliver, Bruno Lepri, Harald Sterly, Renaud Lambiotte, Sébastien Delataille, Marco De Nadai, Emmanuel Letouzé, Albert Ali Salah, Richard Benjamins, Ciro Cattuto, Vittoria Colizza, Nicolas de Cordes, Samuel P. Fraiberger, Till Koebe, Sune Lehmann, Juan Murillo, Alex Pentland, Phuong N Pham, Frédéric Pivetta, Jari Saramäki, Samuel V. Scarpino, Michele Tizzoni, Stefaan Verhulst, and Patrick Vinck. Mobile phone data for informing public health actions across the COVID-19 pandemic life cycle. Science Advances, 2020.

[9] Ramesh Raskar, Isabel Schunemann, Rachel Barbar, Kristen Vilcans, Jim Gray, Praneeth Vepakomma, Suraj Kapa, Andrea Nuzzo, Rajiv Gupta, Alex Berke, et al. Apps gone rogue: Maintaining personal privacy in an epidemic. arXiv preprint arXiv:2003.08567, 2020.

[10] Saheli Roy Choudhury. Singapore says it will make its contact tracing tech freely available to developers.

[11] Kcore Analytics City College of New York. K-core tracing app.

[12] Center for Systems Science and Engineering (CSSE) at Johns Hopkins University. COVID-19 data repository. https://github.com/CSSEGISandData/COVID-19.

[13] Ruoran Li, Caitlin Rivers, Qi Tan, Megan B Murray, Eric Toner, and Marc Lipsitch. The demand for inpatient and ICU beds for COVID-19 in the US lessons from Chinese cities. medRxiv, 2020.

[14] Nicole Winfield. Not a wave, a tsunami, 2020.

[15] Marina Villeneuve and Michael Hill. COVID-19 infections rise in New York with peak weeks away, 2020.

[16] Henrik Salje, Cécile Tran Kiem, Noémie Lefrancq, Noémie Courtejoie, Paolo Bosetti, Juliette Paireau, Alessio Andronico, Nathanaël Hozé, Jehanne Richet, Claire-Lise Dubost, Yann Le Strat, Justin Lessler, Daniel Levy-Bruhl, Arnaud Fontanet, Lulla Opatowski, Pierre-Yves Boelle, and Simon Cauchemez. Estimating the burden of SARS-CoV-2 in France. Science, 2020.

[17] Tung Thanh Le, Zacharias Andreadakis, Arun Kumar, Raúl Gómez Román, Stig Tollefsen, Melanie Saville, and Stephen Mayhew. The COVID-19 vaccine development landscape. Nature Reviews Drug Discovery, 19:305-306, 2020.

[18] Giorgio Guzzetta, Flavia Riccardo, Valentina Marziano, Piero Poletti, Filippo Trentini, Antonino Bella, Xanthi Andrianou, Martina Del Manso, Massimo Fabiani, Stefania Bellino, Stefano Boros, Alberto Mateo Urdiales, Maria Fenicia Vescio, Andrea Piccioli, COVID-19 working 
group, Silvio Brusaferro, Giovanni Rezza, Patrizio Pezzotti, Marco Ajelli, and Stefano Merler. The impact of a nation-wide lockdown on COVID-19 transmissibility in Italy, 2020.

[19] Roy M Anderson, Hans Heesterbeek, Don Klinkenberg, and T Déirdre Hollingsworth. How will country-based mitigation measures influence the course of the COVID-19 epidemic? The Lancet, 395(10228):931-934, 2020.

[20] Joel R Koo, Alex R Cook, Minah Park, Yinxiaohe Sun, Haoyang Sun, Jue Tao Lim, Clarence Tam, and Borame L Dickens. Interventions to mitigate early spread of SARS-CoV-2 in singapore: a modelling study. The Lancet Infectious Diseases, 2020.

[21] Jayson S. Jia, Xin Lu, Yun Yuan, Ge Xu, Jianmin Jia, and Nicholas A. Christakis. Population flow drives spatio-temporal distribution of COVID-19 in China. Nature, 2020.

[22] Juanjuan Zhang, Maria Litvinova, Yuxia Liang, Yan Wang, Wei Wang, Shanlu Zhao, Qianhui Wu, Stefano Merler, Cécile Viboud, Alessandro Vespignani, Marco Ajelli, and Hongjie Yu. Changes in contact patterns shape the dynamics of the COVID-19 outbreak in China. Science, 2020.

[23] Hao-Yuan Cheng, Shu-Wan Jian, Ding-Ping Liu, Ta-Chou Ng, Wan-Ting Huang, and HsienHo Lin. Contact tracing assessment of COVID-19 transmission dynamics in Taiwan and risk at different exposure periods before and after symptom onset. JAMA Internal Medicine, 2020.

[24] Per Block, Marion Hoffman, Isabel J Raabe, Jennifer Beam Dowd, Charles Rahal, Ridhi Kashyap, and Melinda C Mills. Social network-based distancing strategies to flatten the COVID 19 curve in a post-lockdown world. arXiv preprint arXiv:2004.07052, 2020.

[25] Laura Di Domenico, Giulia Pullano, Chiara E. Sabbatini, Pierre-Yves Boëlle, and Vittoria Colizza. Expected impact of lockdown in île-de-France and possible exit strategies. medRxiv, 2020.

[26] Shengjie Lai, Nick W Ruktanonchai, Liangcai Zhou, Olivia Prosper, Wei Luo, Jessica R Floyd, Amy Wesolowski, Chi Zhang, Xiangjun Du, Hongjie Yu, et al. Effect of non-pharmaceutical interventions for containing the COVID-19 outbreak: an observational and modelling study. medRxiv, 2020.

[27] Neil Ferguson, Daniel Laydon, Gemma Nedjati Gilani, Natsuko Imai, Kylie Ainslie, Marc Baguelin, Sangeeta Bhatia, Adhiratha Boonyasiri, ZULMA Cucunuba Perez, Gina CuomoDannenburg, et al. Report 9: Impact of non-pharmaceutical interventions (NPIs) to reduce COVID19 mortality and healthcare demand. Technical report, Imperial College London, 2020. 
[28] Stephen M. Kissler, Christine Tedijanto, Edward Goldstein, Yonatan H. Grad, and Marc Lipsitch. Projecting the transmission dynamics of SARS-CoV-2 through the postpandemic period. Science, 2020.

[29] Shujuan Ma, Jiayue Zhang, Minyan Zeng, Qingping Yun, Wei Guo, Yixiang Zheng, Shi Zhao, Maggie H Wang, and Zuyao Yang. Epidemiological parameters of coronavirus disease 2019: a pooled analysis of publicly reported individual data of 1155 cases from seven countries. medRxiv, 2020.

[30] Tapiwa Ganyani, Cecile Kremer, Dongxuan Chen, Andrea Torneri, Christel Faes, Jacco Wallinga, and Niel Hens. Estimating the generation interval for COVID-19 based on symptom onset data. medRxiv, 2020.

[31] Enrico Lavezzo, Elisa Franchin, Constanze Ciavarella, Gina Cuomo-Dannenburg, Luisa Barzon, Claudia Del Vecchio, Lucia Rossi, Riccardo Manganelli, Arianna Loregian, Nicolò Navarin, et al. Suppression of COVID-19 outbreak in the municipality of Vo, Italy. medRxiv, 2020.

[32] Francesco Pinotti, Laura Di Domenico, Ernesto Ortega, Marco Mancastroppa, Giulia Pullano, Eugenio Valdano, Pierre-Yves Boelle, Chiara Poletto, and Vittoria Colizza. Lessons learnt from 288 COVID-19 international cases: importations over time, effect of interventions, underdetection of imported cases. medRxiv, 2020.

[33] Yang Liu, Rosalind M Eggo, and Adam J Kucharski. Secondary attack rate and superspreading events for SARS-CoV-2. The Lancet, 395(10227):e47, 2020.

[34] Lars Lorch, William Trouleau, Stratis Tsirtsis, Aron Szanto, Bernhard Schölkopf, and Manuel Gomez-Rodriguez. A spatiotemporal epidemic model to quantify the effects of contact tracing, testing, and containment. arXiv preprint arXiv:2004.07641, 2020.

[35] Adam Kucharski, Petra Klepac, Andrew Conlan, Stephen Kissler, Maria Tang, Hannah Fry, Julia Gog, and John Edmunds. Effectiveness of isolation, testing, contact tracing and physical distancing on reducing transmission of SARS-CoV-2 in different settings, 2020.

[36] Robert Hinch, Will Probert, Anel Nurtay, Michelle Kendall, Chris Wymant, Matthew Hall, Katrina Lythgoe, Ana Bulas Cruz, Lele Zhao, Andrea Stewart, Michael Ferretti, Luca Parker, Ares Meroueh, Bryn Mathias, Scott Stevenson, Daniel Montero, James Warren, Nicole K Mather, Anthony Finkelstein, Lucie Abeler-Dörner, and Christophe Bonsall, David Fraser. Effective configurations of a digital contact tracing app: A report to nhsx, 2020. 
[37] European Centre for Disease Prevention and Control. Resource estimation for contact tracing, quarantine and monitoring activities for COVID-19 cases in the eu/eea.

[38] Don Klinkenberg, Christophe Fraser, and Hans Heesterbeek. The effectiveness of contact tracing in emerging epidemics. PLOS ONE, 1(1):1-7, 122006.

[39] Joel Hellewell, Sam Abbott, Amy Gimma, Nikos I Bosse, Christopher I Jarvis, Timothy W Russell, James D Munday, Adam J Kucharski, W John Edmunds, Fiona Sun, Stefan Flasche, Billy J Quilty, Nicholas Davies, Yang Liu, Samuel Clifford, Petra Klepac, Mark Jit, Charlie Diamond, Hamish Gibbs, Kevin [van Zandvoort], Sebastian Funk, and Rosalind M Eggo. Feasibility of controlling COVID-19 outbreaks by isolation of cases and contacts. The Lancet Global Health, 8(4):e488 - e496, 2020.

[40] Timo Smieszek, Stefanie Castell, Alain Barrat, Ciro Cattuto, Peter J White, and Gérard Krause. Contact diaries versus wearable proximity sensors in measuring contact patterns at a conference: method comparison and participants' attitudes. BMC infectious diseases, 16(1):341, 2016.

[41] Rossana Mastrandrea, Julie Fournet, and Alain Barrat. Contact patterns in a high school: a comparison between data collected using wearable sensors, contact diaries and friendship surveys. PloS one, 10(9), 2015.

[42] Laurent Hébert-Dufresne, Benjamin M Althouse, Samuel V Scarpino, and Antoine Allard. Beyond R0: Heterogeneity in secondary infections and probabilistic epidemic forecasting. medRxiv, 2020.

[43] William J Bradshaw, Ethan C Alley, Jonathan H Huggins, Alun L Lloyd, and Kevin M Esvelt. Bidirectional contact tracing is required for reliable COVID-19 control. medRxiv, 2020.

[44] Sadamori Kojaku, Laurent Hébert-Dufresne, and Yong-Yeol Ahn. The effectiveness of contact tracing in heterogeneous networks. arXiv preprint arXiv:2005.02362, 2020.

[45] Gabriel Kaptchuk, Daniel G. Goldstein, Eszter Hargittai, Jake Hofman, and Elissa M. Redmiles. How good is good enough for COVID19 apps? the influence of benefits, accuracy, and privacy on willingness to adopt. arXiv, 2020.

[46] Hossein Gorji, Markus Arnoldini, David F Jenny, Wolf-Dietrich Hardt, and Patrick Jenny. Stecc: Smart testing with contact counting enhances covid-19 mitigation by bluetooth app based contact tracing. medRxiv, 2020. 
[47] Josh A Firth, Joel Hellewell, Petra Klepac, Stephen M Kissler, , Adam J Kucharski, and Lewis G. Spurgin. Combining fine-scale social contact data with epidemic modelling reveals interactions between contact tracing, quarantine, testing and physical distancing for controlling covid-19. medRxiv, 2020.

[48] A. L. Lloyd and R. M. May. How viruses spread among computers and people. Science, 292:1316 - 1317, 2001.

[49] Anna Machens, Francesco Gesualdo, Caterina Rizzo, Alberto E Tozzi, Alain Barrat, and Ciro Cattuto. An infectious disease model on empirical networks of human contact: bridging the gap between dynamic network data and contact matrices. BMC infectious diseases, 13(1):185, 2013.

[50] Piotr Sapiezynski, Arkadiusz Stopczynski, David Dreyer Lassen, and Sune Lehmann. Interaction data from the Copenhagen Networks Study. Scientific Data, 6(315), 2019.

[51] Mathieu Génois and Alain Barrat. Can co-location be used as a proxy for face-to-face contacts? EPJ Data Science, 7(1):11, 2018.

[52] Rossana Mastrandrea, Julie Fournet, and Alain Barrat. Contact patterns in a high school: a comparison between data collected using wearable sensors, contact diaries and friendship surveys. PloS one, 10(9), 2015.

[53] E Rea, J Lafleche, S Stalker, BK Guarda, H Shapiro, I Johnson, SJ Bondy, R Upshur, ML Russell, and M Eliasziw. Duration and distance of exposure are important predictors of transmission among community contacts of ontario sars cases. Epidemiology \& Infection, 135(6):914921, 2007.

[54] Timo Smieszek. A mechanistic model of infection: why duration and intensity of contacts should be included in models of disease spread. Theoretical Biology and Medical Modelling, 6(1):25, 2009.

[55] Vedran Sekara and Sune Lehmann. The strength of friendship ties in proximity sensor data. PloS one, 9(7):e100915, 2014.

[56] Xi He, Eric HY Lau, Peng Wu, Xilong Deng, Jian Wang, Xinxin Hao, Yiu Chung Lau, Jessica Y Wong, Yujuan Guan, Xinghua Tan, et al. Temporal dynamics in viral shedding and transmissibility of COVID-19. Nature Medicine, pages 1-4, 2020.

[57] Juanjuan Zhang, Maria Litvinova, Wei Wang, Yan Wang, Xiaowei Deng, Xinghui Chen, Mei $\mathrm{Li}$, Wen Zheng, Lan Yi, Xinhua Chen, et al. Evolving epidemiology of novel coronavirus 
diseases 2019 and possible interruption of local transmission outside Hubei Province in China: a descriptive and modeling study. medRxiv, 2020.

[58] D Cereda, M Tirani, F Rovida, V Demicheli, M Ajelli, P Poletti, and S Merler. The early phase of the COVID-19 outbreak in lombardy, Italy, 2020.

[59] Ciro Cattuto, Wouter Van den Broeck, Alain Barrat, Vittoria Colizza, Jean-François Pinton, and Alessandro Vespignani. Dynamics of person-to-person interactions from distributed RFID sensor networks. PLOS ONE, 5(7):1-9, 072010.

[60] Hiroshi Nishiura, Tetsuro Kobayashi, Takeshi Miyama, Ayako Suzuki, Sungmok Jung, Katsuma Hayashi, Ryo Kinoshita, Yichi Yang, Baoyin Yuan, Andrei R Akhmetzhanov, et al. Estimation of the asymptomatic ratio of novel coronavirus infections (COVID-19). medRxiv, 2020.

[61] Daniel Oran and Eric Topol. Prevalence of asymptomatic sars-cov-2 infection. Annals of Internal Medicine, 0(0):null, 0. PMID: 32491919.

[62] Qifang Bi, Yongsheng Wu, Shujiang Mei, Chenfei Ye, Xuan Zou, Zhen Zhang, Xiaojian Liu, Lan Wei, Shaun A Truelove, Tong Zhang, et al. Epidemiology and transmission of COVID-19 in Shenzhen China: Analysis of 391 cases and 1,286 of their close contacts. MedRxiv, 2020.

[63] Kenji Mizumoto, Katsushi Kagaya, Alexander Zarebski, and Gerardo Chowell. Estimating the asymptomatic proportion of coronavirus disease 2019 (covid-19) cases on board the diamond princess cruise ship, yokohama, japan, 2020. Eurosurveillance, 25(10):2000180, 2020.

[64] Ying Liu, Albert A Gayle, Annelies Wilder-Smith, and Joacim Rocklöv. The reproductive number of covid-19 is higher compared to sars coronavirus. Journal of travel medicine, 2020.

[65] Julie Fournet and Alain Barrat. Epidemic risk from friendship network data: an equivalence with a non-uniform sampling of contact networks. Scientific reports, 6(1):1-11, 2016.

[66] Juliette Stehlé, Nicolas Voirin, Alain Barrat, Ciro Cattuto, Vittoria Colizza, Lorenzo Isella, Corinne Régis, Jean-François Pinton, Nagham Khanafer, Wouter Van den Broeck, et al. Simulation of an seir infectious disease model on the dynamic contact network of conference attendees. BMC medicine, 9(1):87, 2011.

[67] Yang Liu, Li-Meng Yan, Lagen Wan, Tian-Xin Xiang, Aiping Le, Jia-Ming Liu, Malik Peiris, Leo LM Poon, and Wei Zhang. Viral dynamics in mild and severe cases of covid-19. The Lancet Infectious Diseases, 2020.

[68] Ruiyun Li, Sen Pei, Bin Chen, Yimeng Song, Tao Zhang, Wan Yang, and Jeffrey Shaman. Substantial undocumented infection facilitates the rapid dissemination of novel coronavirus (sars-cov-2). Science, 368(6490):489-493, 2020. 
[69] Quan-Hui Liu, Marco Ajelli, Alberto Aleta, Stefano Merler, Yamir Moreno, and Alessandro Vespignani. Measurability of the epidemic reproduction number in data-driven contact networks. Proceedings of the National Academy of Sciences, 115(50):12680-12685, 2018.

[70] Qun Li, Xuhua Guan, Peng Wu, Xiaoye Wang, Lei Zhou, Yeqing Tong, Ruiqi Ren, Kathy S.M. Leung, Eric H.Y. Lau, Jessica Y. Wong, Xuesen Xing, Nijuan Xiang, Yang Wu, Chao Li, Qi Chen, Dan Li, Tian Liu, Jing Zhao, Man Liu, Wenxiao Tu, Chuding Chen, Lianmei Jin, Rui Yang, Qi Wang, Suhua Zhou, Rui Wang, Hui Liu, Yinbo Luo, Yuan Liu, Ge Shao, Huan $\mathrm{Li}$, Zhongfa Tao, Yang Yang, Zhiqiang Deng, Boxi Liu, Zhitao Ma, Yanping Zhang, Guoqing Shi, Tommy T.Y. Lam, Joseph T. Wu, George F. Gao, Benjamin J. Cowling, Bo Yang, Gabriel M. Leung, and Zijian Feng. Early transmission dynamics in wuhan, china, of novel coronavirus-infected pneumonia. New England Journal of Medicine, 382(13):1199-1207, 2020. PMID: 31995857. 


\section{0 \\ Supplementary Information}

\begin{tabular}{|c|c|c|c|c|}
\hline Name & Inputs & Definition & Description & Source \\
\hline$\omega(\tau)$ & time $\tau$ (days) & $\begin{array}{l}\text { Weibull distribution with shape }= \\
2.826 \text { and scale }=5.665 \text {. }\end{array}$ & $\begin{array}{l}\text { Probability for an infected } \\
\text { individual to transmit the } \\
\text { disease at time } \tau \text {. }\end{array}$ & 1 \\
\hline$R_{0}$ & & $1.2,1.5,2$ & Reproduction number. & $\begin{array}{l}\text { The value } 2 \text { is } \\
\text { taken from the } \\
\text { literature } 64 \text { The } \\
\text { other values rep- } \\
\text { resent scenarios } \\
\text { of new onsets af- } \\
\text { ter a lockdown. }\end{array}$ \\
\hline $\begin{array}{l}\text { Infectiousness } \\
\beta(\tau)\end{array}$ & time $\tau$ (days) & $R_{0} \cdot \omega(\tau)$ & $\begin{array}{l}\text { Infectiousness scaled } \\
\text { with } R_{0} \text {. }\end{array}$ & 1 \\
\hline$\omega_{\text {exposure }}(e)$ & $\begin{array}{l}\text { contact duration } \\
e(\mathrm{sec})\end{array}$ & $\begin{array}{l}1-\left(1-\beta_{0}\right)^{e / \Delta t} \text { with } \Delta t=60 \\
\text { sec and } \beta_{0}=0.002 .\end{array}$ & $\begin{array}{l}\text { Probability for an infected } \\
\text { individual to transmit the } \\
\text { disease given the dura- } \\
\text { tion } e \text { of a contact. }\end{array}$ & $5,65,66$ \\
\hline$\omega_{\text {dist }}\left(s_{s}\right)$ & $\begin{array}{l}\text { signal strength } \\
s_{s}(\mathrm{dBm})\end{array}$ & $\begin{array}{l}1-1 /\left(1+\exp \left(-s \cdot d\left(s_{s}\right)+b\right)\right) \text { with } \\
s=1.5, b=6.6 \text { and } d\left(s_{s}\right)= \\
a /\left(s_{s}+c\right)^{d} \text { with } a=8.851 \cdot 10^{5}, \\
c=113.4, d=3.715\end{array}$ & $\begin{array}{l}\text { Probability for an infected } \\
\text { individual to transmit the } \\
\text { disease given the signal } \\
\text { strength } s_{s} \text { of a contact. }\end{array}$ & $\begin{array}{l}\text { This paper (Sec- } \\
\text { tion A.1. }\end{array}$ \\
\hline$\omega_{\text {data }}\left(\tau, s_{s}, e\right)$ & $\begin{array}{l}\text { time, signal } \\
\text { strength, contact } \\
\text { duration }\end{array}$ & $\omega(\tau) \cdot \omega_{\text {exposure }}(e) \cdot \omega_{\text {dist }}\left(s_{s}\right)$ & $\begin{array}{l}\text { Probability for an infected } \\
\text { individual to transmit the } \\
\text { disease at time } \tau \text {, given } \\
\text { the signal strength } s_{s} \text { and } \\
\text { the duration } e \text { of a con- } \\
\text { tact. }\end{array}$ & This paper. \\
\hline onset time $(\tau)$ & time $\tau$ (days) & $\begin{array}{l}\text { Lognormal distribution with } \mu= \\
1.54, \sigma=0.47, \text { translated by } \\
\text { the delay of } 2 \text { days, and scaled in } \\
{[0, p] \text { with } p=0.8 \text {. }}\end{array}$ & $\begin{array}{l}\text { Probability for an infected } \\
\text { individual to be detected } \\
\text { within time } \tau \text {. }\end{array}$ & 1 \\
\hline$s(\tau)$ & time $\tau$ (days) & $\begin{array}{l}\text { Cumulative distribution of } \\
\text { onset time }(\tau) \text {. }\end{array}$ & $\begin{array}{l}\text { Probability for an infected } \\
\text { individual to be detected } \\
\text { within time } \tau\end{array}$ & 1 \\
\hline
\end{tabular}

Table 3: Characteristic parameters of the disease. 


\section{A Characteristic parameters of the disease}

The infectiousness $\beta(\tau)$ of an infected individual in the continuous model is assumed to be given by the product of $R_{0}$ and the curve $\omega(\tau)$ described in Table 3. For this function we rely on the shape proposed by Ferretti et al.., ${ }^{1}$ which takes into account four different contributions: asymptomatic, pre-symptomatic and symptomatic infectiousness, plus environment transmission representing the indirect contagion occurring for instance via contaminated surfaces. The symptomatic infectiousness has been obtained by Ferretti et al. by making use of generation time data. The pre-symptomatic infectiousness is simply assumed to be equal to the symptomatic one, while the asymptomatic individuals are considered to have only $10 \%$ of the infection potential, in according to the recent literature ${ }^{67 / 68}$ presuming that individuals with no symptoms are generally less contagious. An alternative shape of the curve is discussed in Section A.3.

In the simulation we do not make use of the general infectiousness $\beta(\tau)$ but we consider the contagion probability $\omega(\tau)$ in occasion of each contact. We introduce the dependency of proximity and contact duration, $\omega_{\text {dist }}$ and $\omega_{\text {exposure }}$ respectively, in order to obtain the function $\omega_{\text {data }}$ defined below. The function parameters are adjusted to simulate an epidemics with the correct reproduction number, see Section A.2.

\section{A.1 Definition of the infectiousness as a function of the distance}

The probability $\omega_{\text {dist }}$ for an infected individual to transmit the disease given the signal strength $s_{s}$ of a contact is modelled as follows. We first derive a distribution that expresses the probability of infection as a function of the distance of a contact. It is defined as a sigmoid curve that starts from $100 \%$ infectiousness at zero distance and reaches $0 \%$ infectiousness at 10 meters. The steepness of the curve if determined by additionally requiring that a $50 \%$ infection probability is reached at 4 meters. To express this function in terms of signal strength, we fit a power law to a reference measured distribution. ${ }^{[55}$ The resulting expression of $\omega_{\text {dist }}$ is in Table 3 .

\section{A.2 Parameter tuning to validate the infection probabilities}

Since we couple a continuous model (see Section B in Supplementary Information) with a simulation on a dynamic network (see Section 4.1), we tune the epidemic parameters so that the spreading patterns of the virus are consistent in the two settings. Namely, while the infectiousness $\beta(\tau)$ of the continuous model has an explicit dependence on the reproduction number $R_{0}$ (that 


\begin{tabular}{|c|c|c|}
\hline$\beta_{0}$ & $\operatorname{mean}\left(R_{0}^{\text {data }}\right)$ & $\operatorname{std}\left(R_{0}^{\text {data }}\right)$ \\
\hline 0.0007 & 0.925 & 1.578 \\
\hline 0.0009 & 1.23 & 1.889 \\
\hline $\mathbf{0 . 0 0 1}$ & $\mathbf{1 . 4 7}$ & $\mathbf{2 . 1 5 1}$ \\
\hline 0.0015 & 2.095 & 2.826 \\
\hline 0.002 & 2.26 & 2.857 \\
\hline 0.003 & 3.035 & 4.150 \\
\hline 0.004 & 3.115 & 4.026 \\
\hline 0.005 & 4.32 & 4.808 \\
\hline
\end{tabular}

Table 4: Values of the parameters defining $\omega_{\text {exposure }}$ that have been tested to find a suitable value of $R_{0}^{\text {data }}$, and the corresponding estimates of the mean and standard deviation of $R_{0}^{\text {data }}$. 


\section{A.3 Robustness of the model with respect to the definition of the infec- tiousness probability}

We consider here another infectiousness curve that has been derived in the recent literature by $\mathrm{He}$ et al ${ }^{56}$. We show that, although this curve is different from the curve $\omega$ that we use in this paper, the predictions of the model do not change significantly. This means that the model predictions are robust with respect to changes in the assumed infectiousness curve.

In the work by $\mathrm{He}$ et al. the infectiousness is defined by means of two probability density functions (PDFs): The incubation time $g(t)$ (probability of symptom onset as a function of the time $t$ since infection) and the infectiousness probability $f(t)$ which is a function of the time $t$ elapsed since the symptom onset (and which may be negative, i.e., pre-symptomatic infectiousness). In more details, the function $g$ is in turn taken from Li et al., ${ }^{70}$ and it is a lognormal distribution with mean 1.434065 and std 0.6612 . The function $f$ is instead estimated by He et al..56 it is assumed to be a gamma distribution, and via a max-likelihood approach it is estimated to have shape 2.1157790 and scale 0.6898583 , and to be shifted by an offset 2.3066912. A numerical PDF of the two distributions, computed over $10^{5}$ samples, and the analytical expression of the two PDFs are shown in Figure $6 \mathrm{a}$

From these $g, f$, we can reconstruct a PDF $\omega_{H e}(\tau)$ to be used in our model. This can be done simply by sampling two values from $g$ and $f$ and adding them (the total time from infection to secondary infection is simply split into two intervals separated by the time of symptoms onset). A numerical PDF of this distribution $\omega_{H e}$, computed over the same $10^{5}$ samples, is in Figure 6b. This function $\omega_{H e}$ may also be obtained analytically by convolution as

$$
\omega_{H e}(\tau)=\int_{-\infty}^{\infty} f(\tau-t) g(t) d t,
$$

using the analytically known $f$ and $g$. The discretized convolution is also shown in Figure 6b, and it coincides indeed with the numerical values of $\omega_{H e}$.

Observe that this distribution assigns a small but positive probability (1.42\%, see below) also to infectiousness at negative time (i.e., an individual may infect another one before being itself infected). We ignore this small probability, and we assume that this is due to the fact that the two distributions $f$ and $g$ are estimated from two different populations (according to ${ }^{56}$ ), and thus statistical errors may be present.

Figure 6b shows also the PDF $\omega$ that we used in the paper. Both distributions $\omega$ and $\omega_{H e}$ peak at around 5 days, and they have similar support. The main difference is that the right tail of $\omega_{H e}$ is larger, meaning that it models a non negligible probability of secondary infection also several days after the infection of the spreader. 


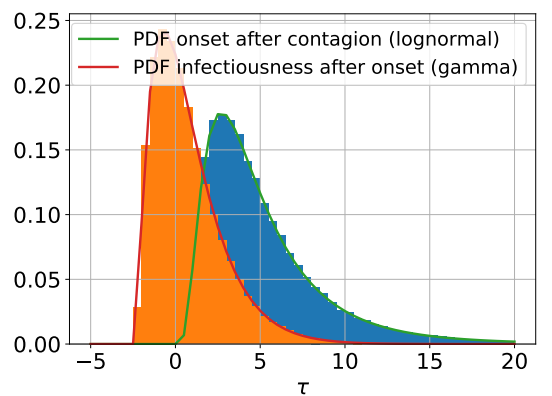

(a)

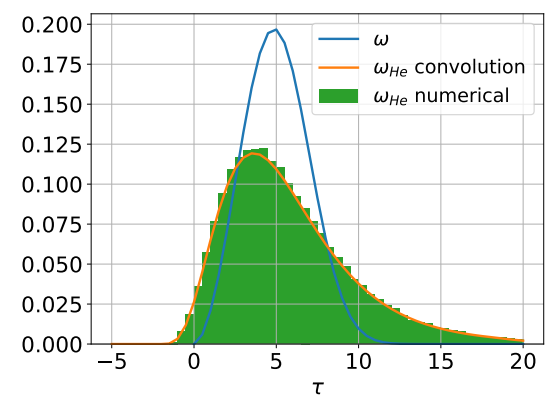

(b)

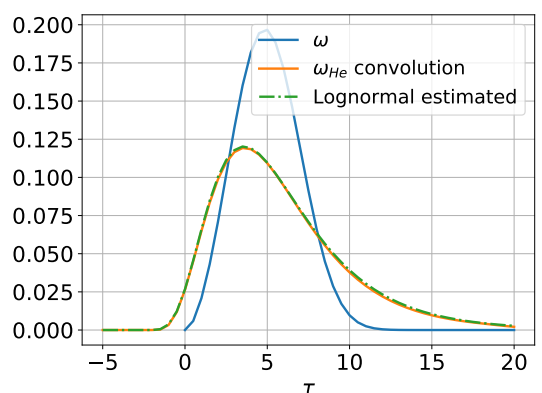

(c)

Figure 6: Visualization and estimation of the infectiousness probability density function (PDF) $\omega_{H} e$. PDFs $f$ and $g$ (Figure 6a); estimated PDF $\omega_{H} e$, and PDF $\omega_{H e}$ (Figure 6b); fir of $\omega_{H e}$ with a lognormal distribution.

To have an analytical expression of $\omega_{H e}$ we try to fit shifted lognormal, gamma, and Weibull distribution to $\omega_{H e}$ (by least-squares minimization over the numerically computed PDF). The best results are obtained with a lognormal distribution with $\mu=2.087, \sigma=0.457$, and shifted by 2.961 , which is plotted in Figure 6c. This allows also to derive an explicit cumulative density function $C D F_{H e}$ of $\omega_{H e}$, which gives an estimate of $C D F_{H e}(0)=0.0142$ (the fraction of negative-time infections).

We can now use this modified infectiousness $\omega_{H e}$ in our model and compare the results with the ones of Figure 4. First, we estimate the parameters defining $\omega_{\text {exposure }}$ as in Section A.2 (see Table 5. The chosen values is also in this case $\beta_{0}=0.001$, corresponding to a value $R_{0}^{\text {data }}=1.5$.

\begin{tabular}{|c|c|c|}
\hline$\beta_{0}$ & mean $\left(R_{0}^{\text {data }}\right)$ & $\operatorname{std}\left(R_{0}^{\text {data }}\right)$ \\
\hline $\mathbf{0 . 0 0 1}$ & $\mathbf{1 . 6 8}$ & $\mathbf{1 . 8 5}$ \\
\hline 0.002 & 2.48 & 2.37 \\
\hline 0.003 & 2.80 & 2.88 \\
\hline 0.004 & 3.13 & 3.13 \\
\hline
\end{tabular}

Table 5: Values of the parameters defining $\omega_{\text {exposure }}$ that have been tested to find a correct value of $R_{0}^{\text {data }}$ for the modified infectiousness probability $\omega_{H e}$, and the corresponding estimates of the mean and standard deviation of $R_{0}^{\text {data }}$.

Using this functional form of $\omega_{H e}$ in the model, we obtain the results of Figure 7 (see Figure 4 for the corresponding results with $\omega$ ). It is clear that the difference is quite limited since only Policy 3 for $\varepsilon_{I}=0.5$ moves from being effective (Figure 4 to being ineffective. Observe that this negligible impact of the change from $\omega$ to $\omega_{H e}$ may be explained by the fact that most contacts in the CNS dataset happen shortly after the contagion (see bottom right panel in Figure 3), and thus the large right tail of $\omega_{H e}$ is not very relevant. We can thus conclude that no significant change in our 


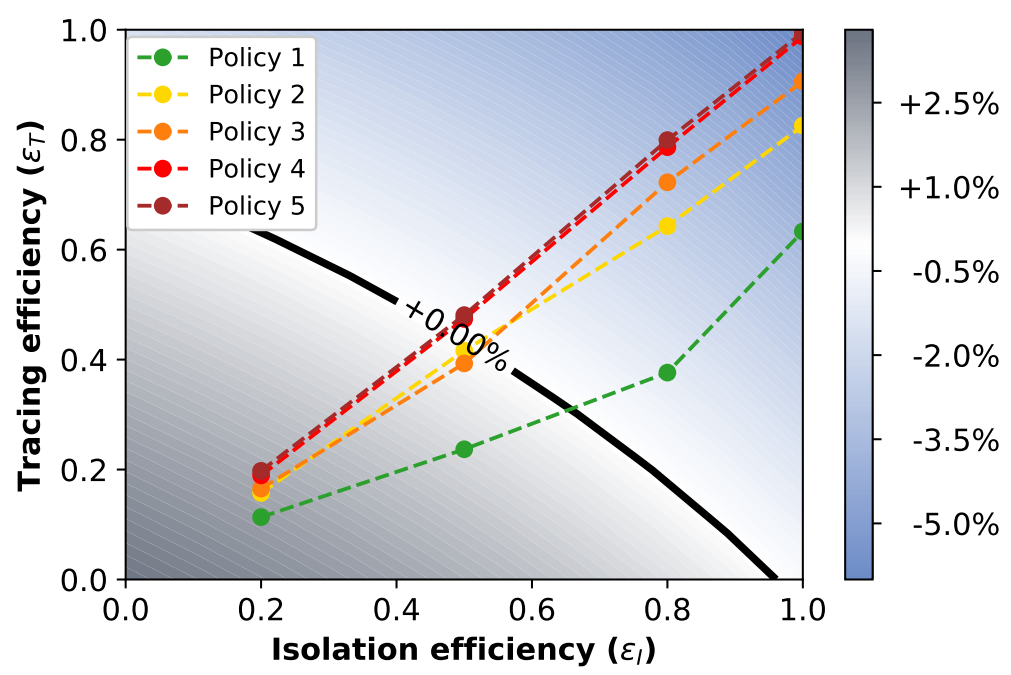

Figure 7: Tracing policy efficiency for alternative infectiousness. Growth or decrease rate of the number of newly infected people using the modified infectiousness curve $\omega_{\mathrm{He}}$. The points correspond to the parameter pairs such that $\varepsilon_{I}$ is an input and $\varepsilon_{T}$ an output of the simulations on real contact data, for the policies of Table 1

Observe that in the absence of containment policies (i.e., $\varepsilon_{I}=\varepsilon_{T}=0$ ) the model predicts a behavior

$$
Y(t, 0, \tau)=\beta(\tau) \int_{\tau}^{t} Y\left(t, \tau, \tau^{\prime}\right) d \tau^{\prime}
$$

In the two cited papers the values of $\varepsilon_{I}, \varepsilon_{T} \in[0,1]$ are fixed, while we assume from now on that they depend on $\tau$. 
i.e., the new infected people are just given by the cumulative number of people who have been infected at previous times, weighted by the infectiousness of the disease. In other words, every previously infected person is a possible agent of new infection, and in this scenario an exponential growth is observed. The isolation and tracing measures, on the other hand, act as discounts on the number of available spreader of the epidemic.

\section{B.1 A more convenient form of the equations}

As mentioned before, the model was analyzed in $n^{1 / 3}$ by considering its asymptotic behavior as $t$ grows to infinity. We instead need a finite-time model that allows a flexible treatment of real data. To this end, it is convenient to use the variable $\Lambda(t, \tau):=Y(t, 0, \tau)\left(\mathrm{se}^{3}\right)$ which represents the number of people which are infected at time $t$ by people who have been infected for time $\tau^{\prime} \leq t$.

With straightforward manipulations, equation (2) can be rewritten for $0 \leq \tau<t$ as follows

$$
\begin{aligned}
Y(t, 0, \tau) & =\beta(\tau)\left(1-\varepsilon_{I}(\tau) s(\tau)\right) \int_{\tau}^{t}\left(1-\varepsilon_{T}(\tau) \frac{s\left(\tau^{\prime}\right)-s\left(\tau^{\prime}-\tau\right)}{1-s\left(\tau^{\prime}-\tau\right)}\right) Y\left(t, \tau, \tau^{\prime}\right) d \tau^{\prime} \\
& =\beta(\tau)\left(1-\varepsilon_{I}(\tau) s(\tau)\right) \int_{0}^{t-\tau}\left(1-\varepsilon_{T}(\tau) \frac{s(\rho+\tau)-s(\rho)}{1-s(\rho)}\right) Y(t, \tau, \rho+\tau) d \rho \\
& =\beta(\tau)\left(1-\varepsilon_{I}(\tau) s(\tau)\right) \int_{0}^{t-\tau}\left(1-\varepsilon_{T}(\tau) \frac{s(\rho+\tau)-s(\rho)}{1-s(\rho)}\right) Y(t-\tau, 0, \rho) d \rho
\end{aligned}
$$

where we changed the integration variable to $\rho:=\tau^{\prime}-\tau$, and we used the translational invariance of $Y$. In the variable $\Lambda$, this reads as

$$
\Lambda(t, \tau)=\beta(\tau)\left(1-\varepsilon_{I}(\tau) s(\tau)\right) \int_{0}^{t-\tau}\left(1-\varepsilon_{T}(\tau) \frac{s(\rho+\tau)-s(\rho)}{1-s(\rho)}\right) \Lambda(t-\tau, \rho) d \rho .
$$

Observe that this is an evolution equation that requires to define an initial number of infected people, i.e., we assume that the quantity $\Lambda(0,0):=\Lambda_{0}$ is a given number.

The quantity of interest is then the total number $\lambda(t):=\int_{0}^{t} \Lambda(t, \tau) d \tau$ of newly infected people at time $t$.

\section{B.2 Discretization}

We fix a value $T>0$ as the maximal simulation time and take $n+1$ points in $[0, T]$ i.e., $\tau_{i}:=i\left(\frac{T}{n}\right)$, $0 \leq i \leq n$.

We will approximate the values of $\Lambda\left(\tau_{k}, \tau_{i}\right)$ for $k=1, \ldots, n$ and $i=0, \ldots, k-1$, while, according to,,$\sqrt[3]{3}$ we set $\Lambda\left(\tau_{k}, \tau_{i}\right)=0$ for all $i \geq k$. Moreover, we assume that the value $\Lambda\left(\tau_{1}, \tau_{0}\right)$ is given. 
Observe that this discretization is equivalent to assume that the number of new cases is measured only at equal discrete times (e.g., at the end of each day) rather than measured continuously.

We show in the next section that the continuous model (3) can be approximated by defining a suitable value for $\Lambda\left(\tau_{1}, \tau_{0}\right)$, and then iteratively computing the values of $\Lambda\left(\tau_{k}, \tau_{i}\right)$ by applying the simple formula

$$
\Lambda\left(\tau_{k}, \tau_{i}\right)=\frac{T}{n} \sum_{j=0}^{k-i-1}\left(A_{\varepsilon_{I}, \varepsilon_{T}}\right)_{i j} \Lambda\left(\tau_{k-i}, \tau_{j}\right), \quad 0 \leq i<k \leq n
$$

where the matrix $A_{\varepsilon_{I}, \varepsilon_{T}} \in \mathbb{R}^{n \times n}$ is defined for $0 \leq i, j \leq n-1$ as

$$
\left(A_{\varepsilon_{I}, \varepsilon_{T}}\right)_{i j}:=\left\{\begin{array}{ll}
\beta\left(\tau_{i}\right)\left(1-\varepsilon_{I}\left(\tau_{i}\right) s\left(\tau_{i}\right)\right)\left(1-\varepsilon_{T}\left(\tau_{j}\right) \frac{s\left(\tau_{j+i}\right)-s\left(\tau_{j}\right)}{1-s\left(\tau_{j}\right)}\right) & \text { if } j \leq n-i-1, \\
0 & \text { if } j>n-i-1,
\end{array},\right.
$$

We remark that this equation is a forward-in-time system, meaning that the computations of the values of $\Lambda(\tau, t)$ is obtained using only values of $\Lambda$ for previous time steps, which have thus already been computed. This is in strong opposition with the case of ${ }^{13}$ where an eigenvalue equation has to be solved, and only the asymptotic state can be estimated.

Moreover, we can use $\Lambda$ to compute

$$
\lambda\left(\tau_{k}\right)=\sum_{i=0}^{k-1} \Lambda\left(\tau_{k}, \tau_{i}\right), \quad 1 \leq k \leq n
$$

\section{B.3 Derivation of the discretization}

We fix a value $T>0$ as the maximal simulation time and take $n+1$ points in $[0, T]$ i.e., $\tau_{i}:=i\left(\frac{T}{n}\right)$, $0 \leq i \leq n$.

The points will be used also to approximate integrals via a right-rectangle quadrature rule, i.e.,

$$
\int_{0}^{\tau_{i}} f(\tau) d \tau \approx \frac{T}{n} \sum_{j=0}^{i-1} f\left(\tau_{j}\right), \quad 1 \leq i \leq n
$$

The goal is to approximate the values of $\Lambda\left(\tau_{k}, \tau_{i}\right)$ for $k=1, \ldots, n$ and $i=0, \ldots, k-1$, while,

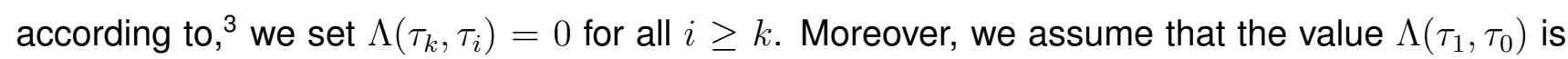
given.

For $1 \leq k \leq n$ we first evaluate (3) at the points, first in the variable $t$ for $\quad 1 \leq k \leq n$, i.e.,

$$
\Lambda\left(\tau_{k}, \tau\right)=\beta(\tau)\left(1-\varepsilon_{I}(\tau) s(\tau)\right) \int_{0}^{\tau_{k}-\tau}\left(1-\varepsilon_{T}(\rho) \frac{s(\rho+\tau)-s(\rho)}{1-s(\rho)}\right) \Lambda\left(\tau_{k}-\tau, \rho\right) d \rho,
$$


and then in the variable $\tau$ for $\tau<t$, that is for $0 \leq i<k \leq n$, i.e.,

$$
\Lambda\left(\tau_{k}, \tau_{i}\right)=\beta\left(\tau_{i}\right)\left(1-\varepsilon_{I}\left(\tau_{i}\right) s\left(\tau_{i}\right)\right) \int_{0}^{\tau_{k}-\tau_{i}}\left(1-\varepsilon_{T}(\rho) \frac{s\left(\rho+\tau_{i}\right)-s(\rho)}{1-s(\rho)}\right) \Lambda\left(\tau_{k}-\tau_{i}, \rho\right) d \rho .
$$

Now observe that for $0 \leq i<k \leq n$ we have

$$
\tau_{k}-\tau_{i}=T\left(\frac{k}{n}\right)-T\left(\frac{i}{n}\right)=T\left(\frac{k-i}{n}\right)=\tau_{k-i},
$$

which ranges between $\tau_{k}$ for $i=0$ and $\tau_{1}$ for $i=k-1$. The last equation becomes for $0 \leq i<k \leq n$

$$
\Lambda\left(\tau_{k}, \tau_{i}\right)=\beta\left(\tau_{i}\right)\left(1-\varepsilon_{I}\left(\tau_{i}\right) s\left(\tau_{i}\right)\right) \int_{0}^{\tau_{k-i}}\left(1-\varepsilon_{T}(\rho) \frac{s\left(\rho+\tau_{i}\right)-s(\rho)}{1-s(\rho)}\right) \Lambda\left(\tau_{k-i}, \rho\right) d \rho .
$$

We can then use the quadrature rule (5) to discretize the integral and obtain

$$
\Lambda\left(\tau_{k}, \tau_{i}\right)=\beta\left(\tau_{i}\right)\left(1-\varepsilon_{I}\left(\tau_{i}\right) s\left(\tau_{i}\right)\right) \frac{T}{n} \sum_{j=0}^{k-i-1}\left(1-\varepsilon_{T}\left(\tau_{j}\right) \frac{s\left(\tau_{j}+\tau_{i}\right)-s\left(\tau_{j}\right)}{1-s\left(\tau_{j}\right)}\right) \Lambda\left(\tau_{k-i}, \tau_{j}\right) .
$$

Observe that the upper limit in the sum has values $0 \leq k-i-1 \leq k-1$ for $0 \leq i<k$. Moreover, in this case we have for $0 \leq j \leq k-i-1$ that

$$
\tau_{j}+\tau_{i}=T\left(\frac{j}{n}\right)+T\left(\frac{i}{n}\right)=T\left(\frac{j+i}{n}\right)=\tau_{j+i},
$$

which ranges between $\tau_{i}$ and $\tau_{k-1}$. Inserting this into the last equation we get for $0 \leq i<k \leq n$

$$
\begin{aligned}
\Lambda\left(\tau_{k}, \tau_{i}\right) & =\beta\left(\tau_{i}\right)\left(1-\varepsilon_{I}\left(\tau_{i}\right) s\left(\tau_{i}\right)\right) \frac{T}{n} \sum_{j=0}^{k-i-1}\left(1-\varepsilon_{T}\left(\tau_{j}\right) \frac{s\left(\tau_{j+i}\right)-s\left(\tau_{j}\right)}{1-s\left(\tau_{j}\right)}\right) \Lambda\left(\tau_{k-i}, \tau_{j}\right) \\
& =\frac{T}{n} \sum_{j=0}^{k-i-1} \beta\left(\tau_{i}\right)\left(1-\varepsilon_{I}\left(\tau_{i}\right) s\left(\tau_{i}\right)\right)\left(1-\varepsilon_{T}\left(\tau_{j}\right) \frac{s\left(\tau_{j+i}\right)-s\left(\tau_{j}\right)}{1-s\left(\tau_{j}\right)}\right) \Lambda\left(\tau_{k-i}, \tau_{j}\right) .
\end{aligned}
$$

We can define the matrix $A_{\varepsilon_{I}, \varepsilon_{T}} \in \mathbb{R}^{n \times n}$ whose entries are defined for $0 \leq i, j \leq n-1$ as

$$
\left(A_{\varepsilon_{I}, \varepsilon_{T}}\right)_{i j}:=\left\{\begin{array}{ll}
\beta\left(\tau_{i}\right)\left(1-\varepsilon_{I}\left(\tau_{i}\right) s\left(\tau_{i}\right)\right)\left(1-\varepsilon_{T}\left(\tau_{j}\right) \frac{s\left(\tau_{j+i}\right)-s\left(\tau_{j}\right)}{1-s\left(\tau_{j}\right)}\right) & \text { if } j \leq n-i-1, \\
0 & \text { if } j>n-i-1,
\end{array},\right.
$$

which has a triangular structure (the first row is nonzero, in the second row the last element is zero, $\ldots$, in the last row only the first element is nonzero).

With this matrix we can rewrite (6) as

$$
\Lambda\left(\tau_{k}, \tau_{i}\right)=\frac{T}{n} \sum_{j=0}^{k-i-1}\left(A_{\varepsilon_{I}, \varepsilon_{T}}\right)_{i j} \Lambda\left(\tau_{k-i}, \tau_{j}\right), \quad 0 \leq i<k \leq n
$$

which is a recursive equation that determines the evolution of $\Lambda(t, \tau)$ once an initial condition is given. 
The only remaining case is $i=0$, and in this case the formula (7) gives instead

$$
\begin{aligned}
\Lambda\left(\tau_{k}, \tau_{0}\right) & =\frac{T}{n} \sum_{j=0}^{k-1}\left(A_{\varepsilon_{I}, \varepsilon_{T}}\right)_{0 j} \Lambda\left(\tau_{k}, \tau_{j}\right) \\
& =\frac{T}{n}\left(A_{\varepsilon_{I}, \varepsilon_{T}}\right)_{00} \Lambda\left(\tau_{k}, \tau_{0}\right)+\frac{T}{n} \sum_{j=1}^{k-1}\left(A_{\varepsilon_{I}, \varepsilon_{T}}\right)_{0 j} \Lambda\left(\tau_{k}, \tau_{j}\right)
\end{aligned}
$$

thus

$$
\Lambda\left(\tau_{k}, \tau_{0}\right)=\left(1-\frac{T}{n}\left(A_{\varepsilon_{I}, \varepsilon_{T}}\right)_{00}\right)^{-1} \frac{T}{n} \sum_{j=1}^{k-1}\left(A_{\varepsilon_{I}, \varepsilon_{T}}\right)_{0 j} \Lambda\left(\tau_{k}, \tau_{j}\right)
$$

where

$$
\begin{aligned}
\left(A_{\varepsilon_{I}, \varepsilon_{T}}\right)_{00} & =\beta\left(\tau_{0}\right)\left(1-\varepsilon_{I}\left(\tau_{0}\right) s\left(\tau_{0}\right)\right)\left(1-\varepsilon_{T}\left(\tau_{0}\right) \frac{s\left(\tau_{0}\right)-s\left(\tau_{0}\right)}{1-s\left(\tau_{0}\right)}\right) \\
& =\beta\left(\tau_{0}\right)\left(1-\varepsilon_{I}\left(\tau_{0}\right) s\left(\tau_{0}\right)\right)
\end{aligned}
$$

and thus

$$
\begin{aligned}
\left(1-\frac{T}{n}\left(A_{\varepsilon_{I}, \varepsilon_{T}}\right)_{00}\right)^{-1} \frac{T}{n} & =\frac{T}{n-T\left(A_{\varepsilon_{I}, \varepsilon_{T}}\right)_{00}} \\
& =\frac{T}{n-T \beta\left(\tau_{0}\right)\left(1-\varepsilon_{I}\left(\tau_{0}\right) s\left(\tau_{0}\right)\right)} .
\end{aligned}
$$

This term is positive if and only if

$$
0<n-T \beta\left(\tau_{0}\right)\left(1-\varepsilon_{I}\left(\tau_{0}\right) s\left(\tau_{0}\right)\right) \Rightarrow \beta\left(\tau_{0}\right)\left(1-\varepsilon_{I}\left(\tau_{0}\right) s\left(\tau_{0}\right)\right)<n / T .
$$

Since the left hand side is at most $\beta\left(\tau_{0}\right)$, it is sufficient to require that $n / T>\beta\left(\tau_{0}\right)$, or $n>\beta\left(\tau_{0}\right) \cdot T$. In this way we defined $\Lambda\left(\tau_{k}, \tau_{i}\right)$ for all values $1 \leq k \leq n$ and $0 \leq i<k$. It remains to assign the value $\Lambda\left(\tau_{1}, \tau_{0}\right)$, which can be fixed to the initial value $\Lambda_{0}$.

\section{Evaluation of additional containment measures and refined policies}

Some extensions are possible to our current setting, and we report the most relevant ones in the following. Each consists in additional steps to either enhance an existing policy or to replace it in order to investigate the effect of other important aspects of the tracing procedure. 


\section{C.1 Longer and shorter tracing memory}

The robustness of the tracing policies is also of fundamental interest, and indeed we introduced five different policies (Table 1) that cover a wide range of possible sensitivity levels, both in space and in time.

It remains to explore how these policies depend on the memory length of the contact history, which has been set to 7 days in all the previous simulations (see Section 2).

First, it is interesting to understand whether or not an increased memory would improve the effectiveness of each policy. We thus repeat the experiments assuming that the contacts of each individual are recorded for 10 days in the past, and report the results in Figure 8a. When compared with the original setting (see Figure 4), it is clear that the increased memory brings a negligible advantage (at the price of increased storage requirements). Indeed, the only visible improvement is a slight increase of the tracing effectiveness of Policy 4, which is now essentially equivalent to Policy 5.

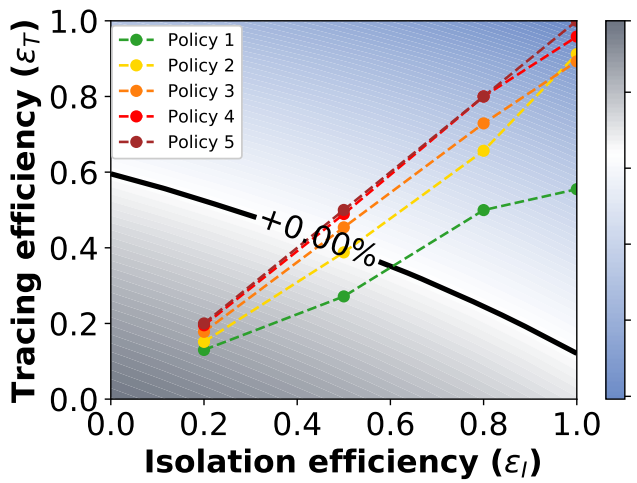

(a)
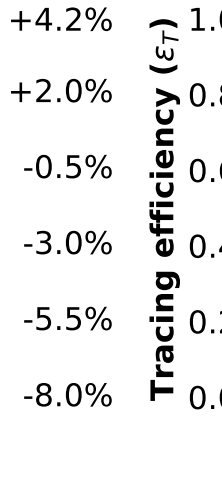$$
\text { Isolat }
$$

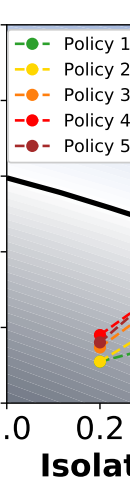

Figure 8: Tracing policy efficiency with longer or shorter contact memory. Growth or decrease rate of the number of newly infected people and efficiency of the containment policies assuming different time lengths of the contact tracing memory. Long memory in Figure 8a where, in addition to the basic setting, the app keeps track of 10 days (instead of 7 days) of past contacts; short memory in Figure 8b where the app keeps track of only 4 days of past contacts.

Second, it is worth investigating if a shorter tracing memory would give improvements in terms of the numbers of false negatives. We thus repeat the simulations assuming that the memory is reduced instead to 4 days, still including the 2 days delay in the case reporting as in all other settings. The results are are in Figure $8 \mathrm{~b}$, and it is clear that the shorter memory reduces, even if only slightly, the effectiveness of the policies. On the other hand, Figure 9 shows that the number 
of false positive is not significantly reduced with respect to the 7 days memory case (Figure 5), and thus it appears to be of no benefit in this setting.

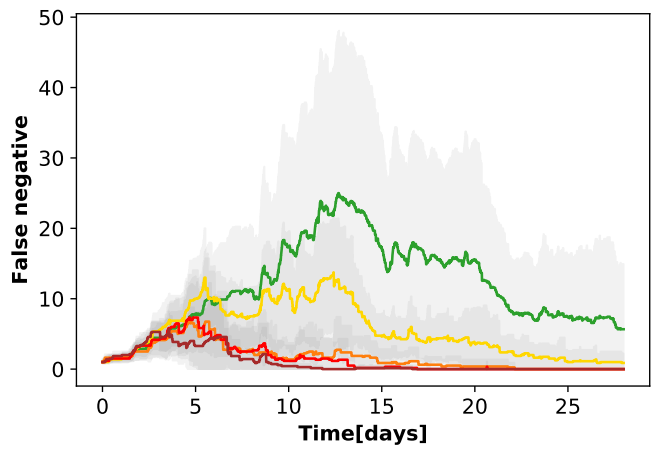

(a)

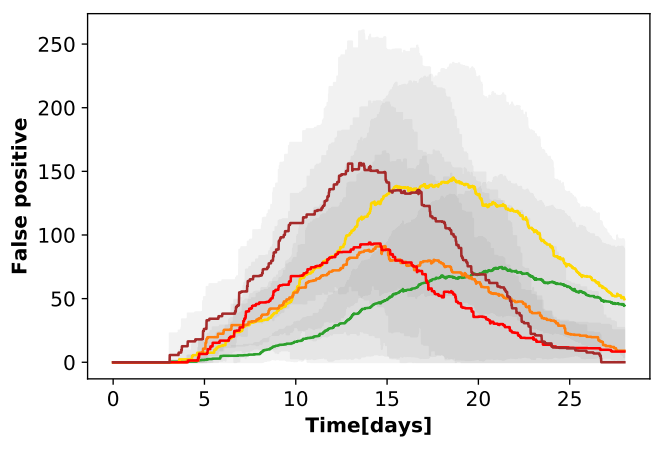

(b)

\begin{tabular}{|c|c|c|}
\hline & $\begin{array}{c}\text { Total } \\
\text { quarantines }\end{array}$ & $\begin{array}{c}\text { Attack } \\
\text { rate }\end{array}$ \\
\hline$\bullet$ & $182 \pm 128$ & $0.24 \pm 0.06$ \\
\hline$\bullet$ & $208 \pm 131$ & $0.12 \pm 0.04$ \\
\hline$\bullet$ & $170 \pm 110$ & $0.08 \pm 0.02$ \\
\hline$\bullet$ & $181 \pm 140$ & $0.063 \pm 0.02$ \\
\hline$\bullet$ & $250 \pm 123$ & $0.046 \pm 0.01$ \\
\hline
\end{tabular}

Figure 9: False positive and negative in quarantines with reduced contact memory. Temporal evolution of the numbers of false negatives, i.e. infected individuals not quarantined, (Figure 9a and false positives, i.e. not infected individuals quarantined, (Figure 9b) for the five different policies, assuming an isolation efficiency of $\varepsilon_{I}=0.8$ and a reduced tracing memory ( 4 days). The graphs report the mean and standard deviation (shading) over 20 independent runs. The table reports mean and standard deviation of the total number of distinct individuals who have been quarantined over the whole simulation timeline and the percentage of those among them who were effectively infected (true positive), corresponding to the attack rate.

\section{C.2 Second order tracing}

An additional possibility is to keep track of contacts in a recursive way. Namely, when an individual is isolated, not only its contacts are quarantined, but also its contacts' contacts. This obviously means an enhanced risk in terms of preserving the privacy of individuals, and hence the major open question regarding this kind of policies is whether or not the increased intrusiveness into an individual's social network provides a tangible improvement of the virus containment efforts.

A complete study of this scenario is beyond the scope of this paper for two reasons. First, the continuous model (see Section B) does not take into consideration this kind of tracing, and there is thus no way to use in a meaningful way any information provided by the study of the dataset. Second, although the datasets we consider are the state of the art in interaction monitoring, they are still rather small to study in a realistic way the effect of a policy that rapidly traces a very large number of persons.

Nevertheless, we find meaningful to report here a preliminary study of this additional, more intru- 
sive tracing policy. We simulated the epidemic on the Copenhagen Study dataset with one random initial infected individual (the same initial condition that we used for the other cases). We observe that after some time steps (depending on $\varepsilon_{I}$ ) the patient zero is identified and isolated, while its contacts are traced. However in most of the cases the patient zero was not able to transmit the virus to other individuals, or in alternative very few people have been infected. In any case, a large part of the population is immediately quarantined, largely decreasing the basin of susceptible people and therefore stopping the spreading. The end of the epidemic is reached at the cost of quarantining many not infected people, in average: $3 \%$ of the population with Policy $1,6 \%$ with Policy $2,7 \%$ with Policy 3, 9\% with Policy 4 and $11 \%$ with Policy 5 . This results in an invasive procedure with a large amount of false positives and a negligible number of true positives, in agreement with the results obtained by Firth et al. ${ }^{47}$

We remark once more that the reliability of this result is limited, being linked to a specific dataset and not to a general theory. Our insight is that the efficiency of the procedure is due to the finite dimension of the agent sample, where such an immediate and preventive intervention is sufficient to cut all the links between infected and susceptible, resulting in a situation which is more akin to a lock-down than to actual contact tracing.

Moreover, we should mention the fact that we did not consider the effect of a lower app adoption in this case, assuming that $100 \%$ of the population can be correctly traced. Such a high level of tracing is even more unrealistic when considering second order tracing, as a large compliance is obtainable only if privacy is perfectly preserved. ${ }^{45}$

For these reasons we remark that the concept of second-order tracing, a topic of recent discussions, deserves further investigation and may possibly be expanded in a follow-up of this work.

\section{C.3 Effect of a reduced app adoption}

To complement the analysis of Section 2.3 on the effect of a limited app adoption, we provide here a numerical quantification of the reduction in the values of $\varepsilon_{T}$. For the five policies of Table 1 and for $\varepsilon_{I}=1$, we report in Table 6 the ratio between the values of $\varepsilon_{T}$ in the case of a limited app adoption $(60 \%$ or $80 \%)$ and in the case of full app adoption (100\%), in all cases with $R_{0}=1.5$. Observe that the computed values are actually quite close to show a quadratic reduction effect.

\section{C.4 Variations in the number of asymptomatic individuals}

To additionally verify the robustness of our predictions with respect to the epidemiological modelling, we assume here that the number of asymptomatic individuals is $20 \%$, and additionally that a 


\begin{tabular}{|c|c|c|c|c|c|}
\hline App adoption & $\bullet$ Policy 1 & $\bullet$ Policy 2 & $\bullet$ Policy 3 & $\bullet$ Policy 4 & $\bullet$ Policy 5 \\
\hline $60 \%$ & $42.6 \%$ & $53.6 \%$ & $45.6 \%$ & $30.8 \%$ & $45.3 \%$ \\
\hline $80 \%$ & $48.8 \%$ & $85.1 \%$ & $77.7 \%$ & $69.3 \%$ & $72.0 \%$ \\
\hline
\end{tabular}

Table 6: Ratio of the values of $\varepsilon_{T}$ between a partial and a full app adoption, for the five policies of Tabele 1 and $\varepsilon_{I}=0.8$. The values are rounded to the first decimal digit.

randomized testing policy that covers $25 \%$ of the asymptomatic population is in place. In this case, our results (see Figure 10) show that the policies of Table 1 would be more effective. Especially, in this setting Policy 2 is successful in containing the spread of the virus even for $\varepsilon_{I}=0.8$, differently from the more pessimistic case of $40 \%$ asymptomatics.

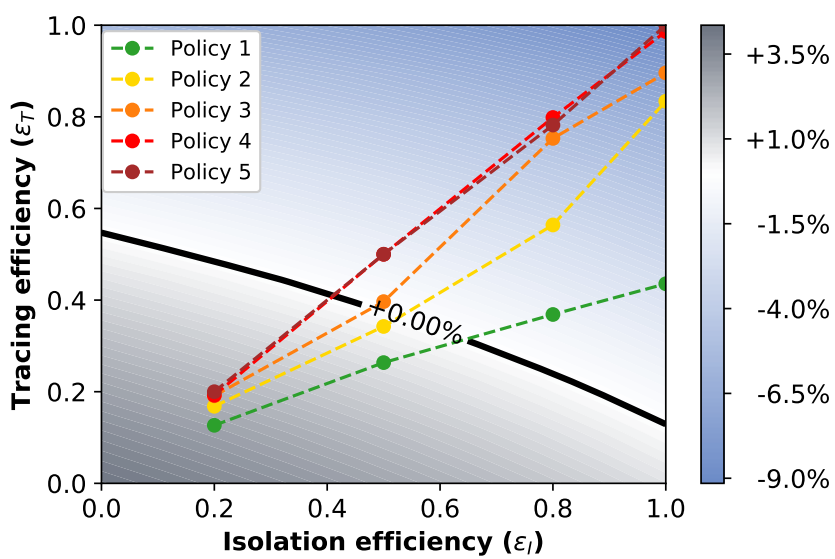

Figure 10: Tracing policy efficiency with $\mathbf{8 0} \%$ asymptomatic and $\mathbf{2 5} \%$ random testing. Growth or decrease rate of the number of newly infected people assuming that symptomatic people account for the $80 \%$ of the infected individuals, that they can be isolated and that an additional $25 \%$ of asymptomatic can be identified via randomized testing. The points correspond to the parameter pairs such that $\varepsilon_{I}$ is an input and $\varepsilon_{T}$ an output of the simulations on real contact data, for the policies of Table 1 .

\section{C.5 Close-range short-exposure vs long-range long-exposure interactions}

We test here two additional policies obtained by mixing a low space resolution and a high time resolution, and viceversa. The policies are defined in Table 7. Policy 6 has a signal strength of $-70 \mathrm{dBm}$ and a duration of 10 minutes, resulting in a policy that captures short exposure but close range interactions. Policy 7 instead captures long exposure but long range interactions, having a threshold signal strength of $-91 \mathrm{dBm}$ and a duration of 30 minutes. 


\begin{tabular}{|c|c|c|c|}
\hline ID & $\begin{array}{c}\text { Signal strength } \\
(\mathrm{dBm})\end{array}$ & $\begin{array}{c}\text { Duration } \\
(\mathrm{min})\end{array}$ & Fraction \\
\hline - Policy 6 & -70 & 10 & $17.9 \%$ \\
\hline - Policy 7 & -91 & 30 & $2.1 \%$ \\
\hline
\end{tabular}

Table 7: Parameters defining the two additional policies, and fraction of the total number of interactions of the CNS dataset that they are able to detect.

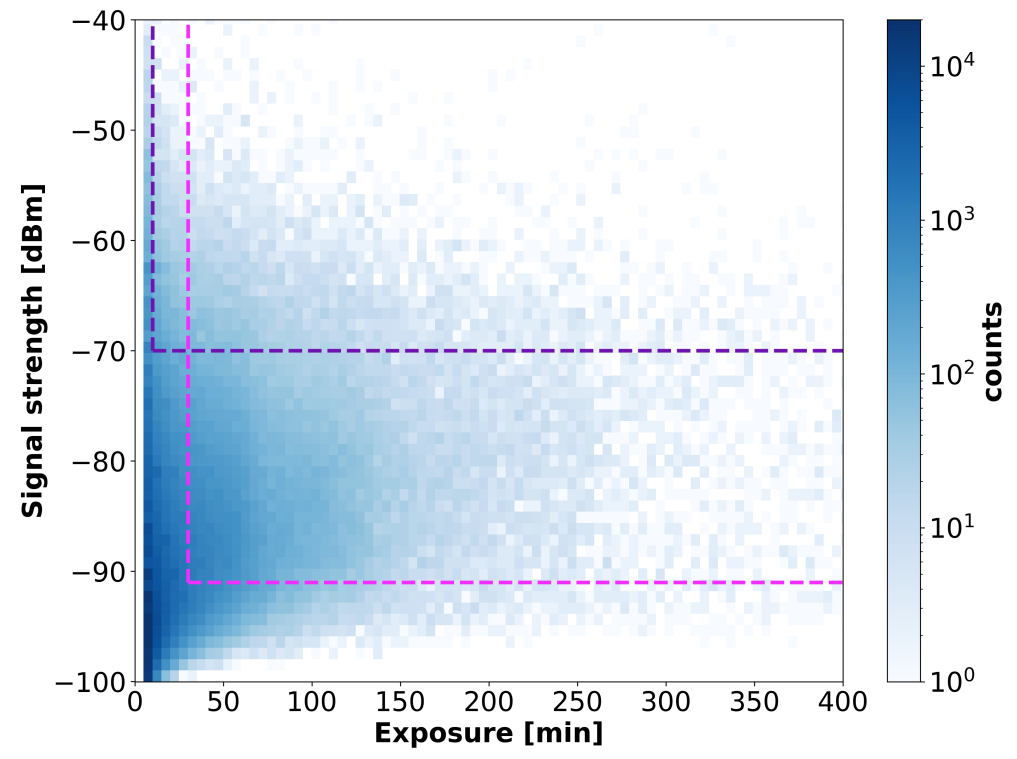

(a)

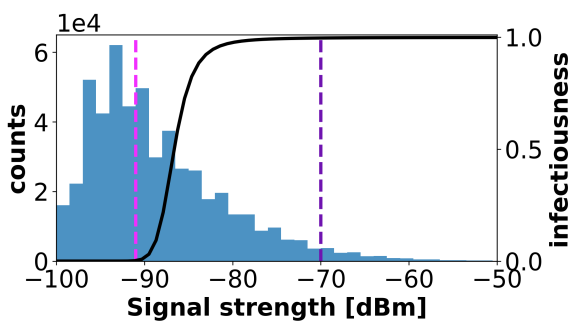

(b)

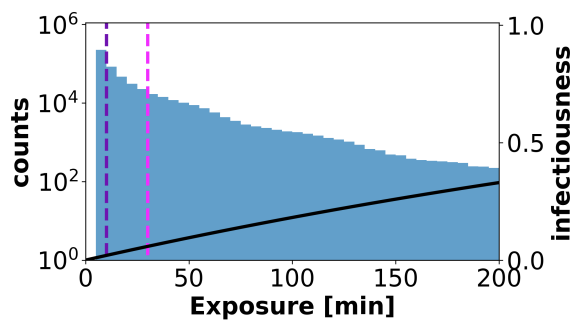

(c)

Figure 11: Distribution of the duration (Figure 11c) and signal strength (taken as a proxy for proximity, Figure 11b of the contacts in the CNS dataset. Figure 11a gives a scatterplot of signal strength vs duration, and displays the thresholds defining the two policies of Table 7 .

The values of the parameters $\left(\varepsilon_{I}, \varepsilon_{T}\right)$ characterizing the numerical simulations for the new policies with $R_{0}=1.5$ are shown in Figure 12 (see Figure 4, center-right panel, for a comparison with the policies of Table 1), and it is clear that Policy 7 is as effective as the most restrictive policies (Policy 4 and Policy 5), while Policy 6 fails to contain the virus for an isolation efficiency smaller than 0.8. The efficiency of quarantines is assessed by the number of false positives and false negatives, reported in Figure 13.

We deduce that the ability to control the contagion seems to be more sensitive to duration of con- 
tacts than to their spatial distance. Indeed, policies which capture close range but short exposure interactions happen to be less performative in quarantining people than those signaling long range interactions with long exposure. In other words, quarantining individuals who have had a short interaction with an infected one, even if at close-range, is unnecessary. On the other hand, it appears to be important to track contacts with a high spatial resolution, including the ones that happens at a rather long distance.

However, we remark once more that these results are depending on the infectiousness model that we have defined here, and that they could possibly change in a different setting.

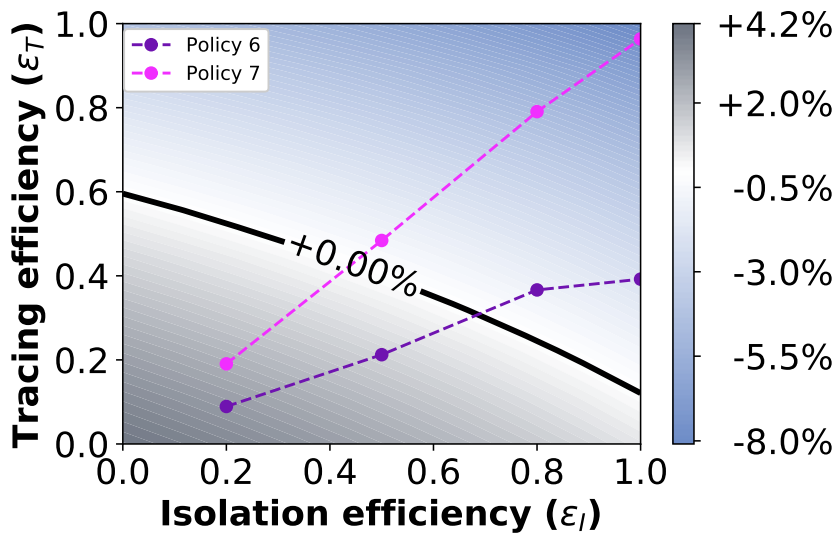

Figure 12: Tracing policy efficiency (alternative policies). Growth or decrease rate of the number of newly infected people assuming that symptomatic people can be isolated and that an additional $50 \%$ of asymptomatic cases can be identified via randomized testing. The points correspond to the parameter pairs such that $\varepsilon_{I}$ is an input and $\varepsilon_{T}$ an output of the simulations on real contact data, for the policies of Table 7 .

Figures 14 and 15 refer to the more realistic case where the app adoption is reduced to $80 \%$. We also maintaing the assumption that $20 \%$ of the infected individuals are asymptomatics or, equivalently, that they are instead the $40 \%$ and an additional $20 \%$ (that is $50 \%$ of the asymptomatics) is identified through random testing.

\section{Extended results on SocioPatterns datasets}

In this section we present the results of simulations performed on two different datasets: (i) High_School13, ${ }^{52}$ collected in a French high school, and (ii) InVS15, ${ }^{51}$ collected in a French workplace. Both datasets have been collected using the sensing platform developed by the SocioPat- 


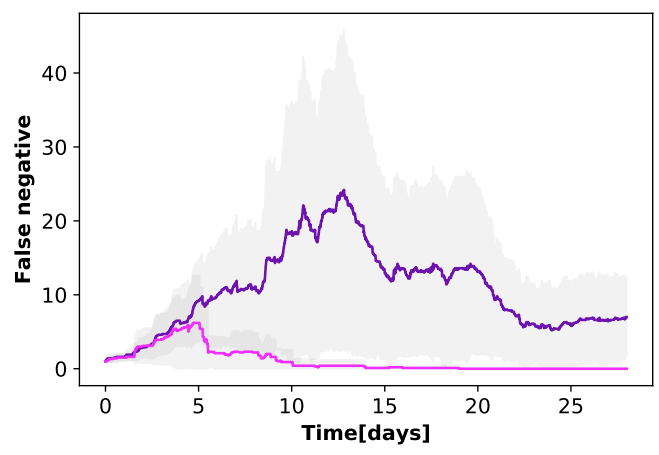

(a)

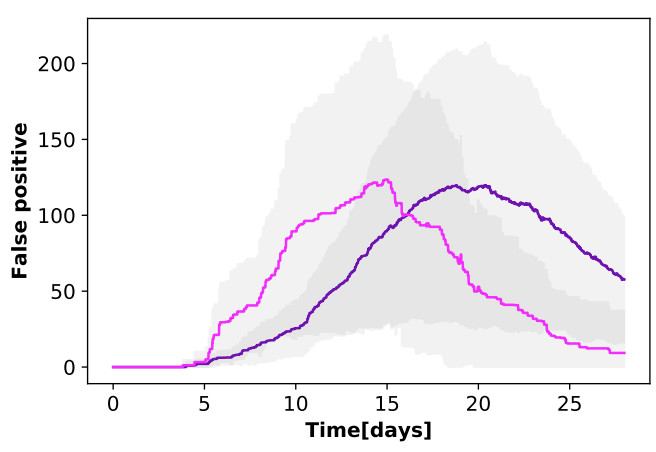

(b)

\begin{tabular}{|c|c|c|}
\hline & $\begin{array}{c}\text { Total } \\
\text { quarantines }\end{array}$ & $\begin{array}{c}\text { Attack } \\
\text { rate }\end{array}$ \\
\hline - & $241 \pm 121$ & $0.186 \pm 0.03$ \\
\hline$\bullet$ & $184 \pm 121$ & $0.059 \pm 0.02$ \\
\hline
\end{tabular}

Figure 13: False positive and negative in quarantines (alternative policies). Temporal evolution of the numbers of false negatives (Figure 13a) and false positives (Figure 13b) for the policies of Table 7, assuming an isolation efficiency of $\varepsilon_{I}=0.8$. The graphs report the mean and standard deviation (shading) over 20 independent runs. The table reports mean and standard deviation of the total number of distinct individuals who have been quarantined over the whole simulation timeline and the percentage of those among them who were effectively infected (true positive), corresponding to the attack rate.

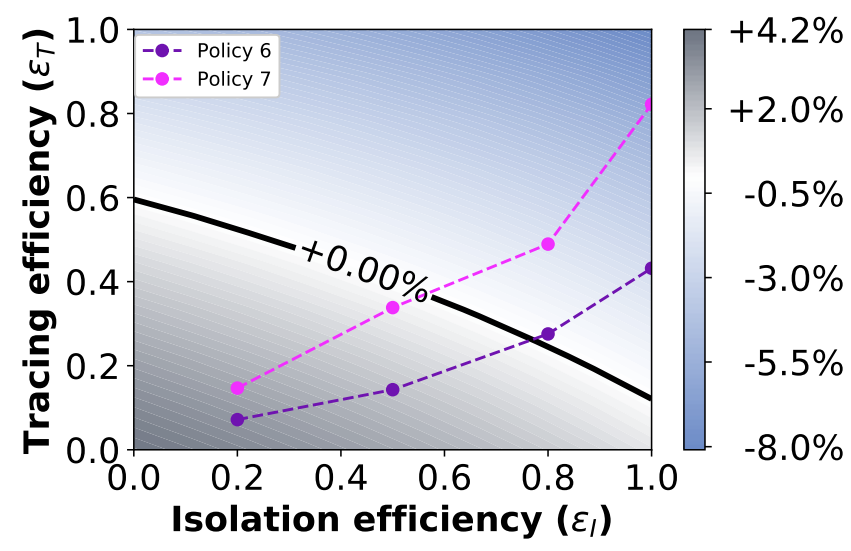

Figure 14: Tracing policy efficiency with $\mathbf{8 0 \%}$ symptomatic (alternative policies). Growth or decrease rate of the number of newly infected people assuming an $80 \%$ app adoption level, with $80 \%$ symptomatics. The points correspond to the parameter pairs such that $\varepsilon_{I}$ is an input and $\varepsilon_{T}$ an output of the simulations on real contact data, for the policies of Table 7 .

terns collaboration 1 , which is based on wearable active Radio Frequency Identification (RFID) devices that exchange radio packets, detecting close proximity $(\leq 1.5 \mathrm{~m})$ of individuals wearing the devices. .59 These data do not contain information on the signal strength, but simply give a list of contacts between individuals with a resolution of 20 seconds. Both simulations and policies are

${ }^{1}$ http://www.sociopatterns.org/ 


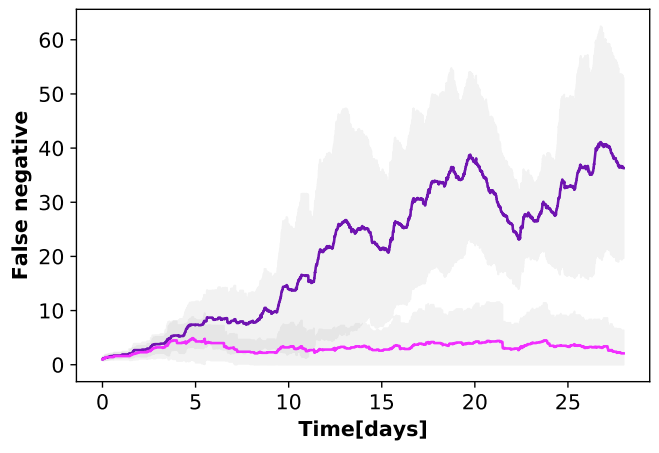

(a)

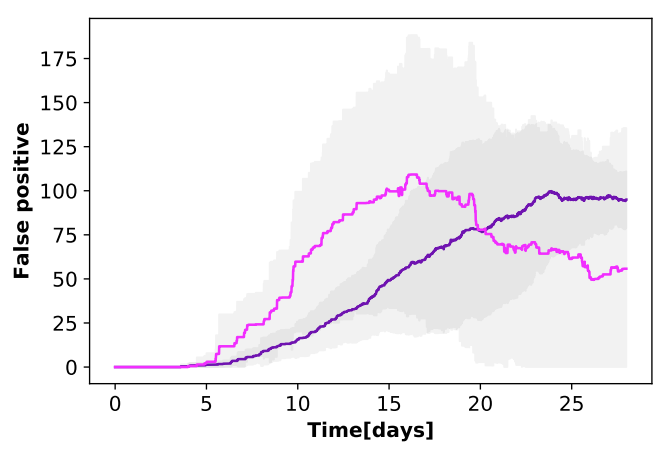

(b)

\begin{tabular}{|c|c|c|}
\hline & $\begin{array}{c}\text { Total } \\
\text { quarantines }\end{array}$ & $\begin{array}{c}\text { Attack } \\
\text { rate }\end{array}$ \\
\hline$\bullet$ & $186 \pm 81$ & $0.23 \pm 0.06$ \\
\hline$\bullet$ & $208 \pm 132$ & $0.066 \pm 0.03$ \\
\hline
\end{tabular}

Figure 15: False positive and negative in quarantines with $\mathbf{8 0} \%$ app adoption (alternative policies). Temporal evolution of the numbers of false negative (Figure 15a) and false positive (Figure 15b) for the policies of Table 7, assuming an isolation efficiency of $\varepsilon_{I}=0.8$, an $80 \%$ app adoption level, and with $80 \%$ symptomatics. The graphs depict the mean and standard deviation over 20 independent runs. The table reports mean and standard deviation of the total number of distinct individuals who have been quarantined over the whole simulation timeline and the percentage of those among them who were effectively infected (true positive), corresponding to the attack rate.

thus defined only as a function of contact durations.

In order to see the effectiveness of the policies and the spreading of the virus, it is needed that the length of the collected data is larger than 15 days. As the SocioPatterns data have a high temporal resolution (20 seconds) but were collected for shorter overall durations, we artificially extend the length of each dataset by replicating it (coping and pasting the entire dataset at the end of the dataset itself). Table 8 gives the number of nodes, the length of the dataset (in days) and the duration of the replicated data.

\begin{tabular}{|l|c|c|}
\hline & InVS15 & High_School13 \\
\hline \# of nodes & 211 & 320 \\
\hline Days & 11.5 & 4.2 \\
\hline Extended Days & 46 & 16.8 \\
\hline
\end{tabular}

Table 8: Number of nodes, days and extended days for each SocioPatterns dataset.

For both these datasets, similarly to the CNS dataset, most contacts happen before the infectiousness reaches its peak (Figure 16), even if contacts are present for all possible durations. Nevertheless, these are sufficient to spread the infection.

We further run the simulations on the network for the five policies of Table 1 (recall that only 


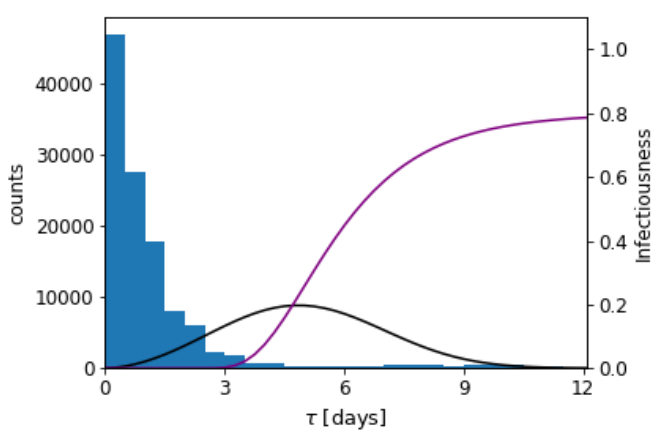

(a)

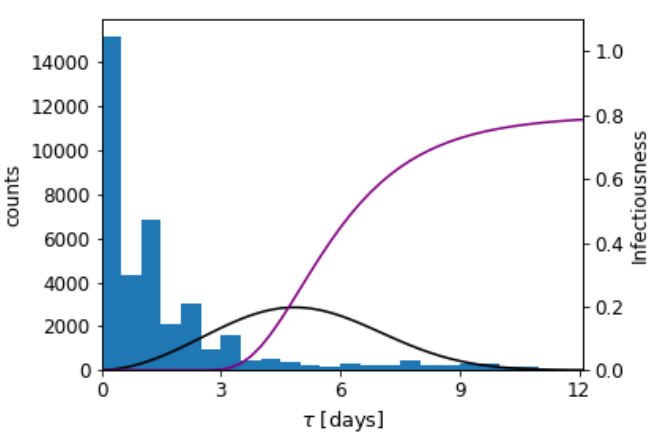

(b)

Figure 16: Distribution of the time since infection of the people having contacts, probability distribution $\omega(\tau)$ (black line) determining the infectiousness as a function of time, and distribution $s(\tau)$ determining the cumulative probability to detect an infected person (purple line). The two plots are obtained with $\varepsilon_{I}=0.8$ and Policy 5 for the InVS15 (Figure 16a) and the High_School13 datasets (Figure 16b).

distances are taken into account). Similarly to the case of the CNS dataset (Figure 4), all policies realize containment if $\varepsilon_{I}$ is at least 0.8 (Figure 17). Additionally, for the $\operatorname{lnVS} 15$ dataset all policies except Policy 1 are effective also for $\varepsilon_{I}=0.5$ (Figure 17a).

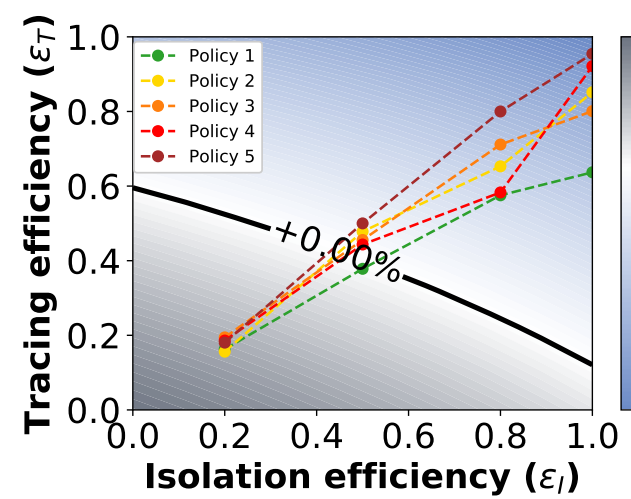

(a)
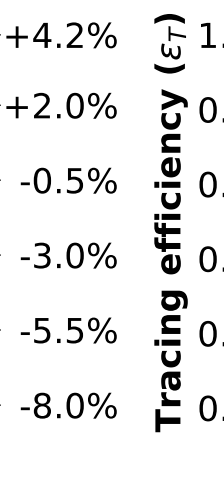

$+4.2 \%$

$+2.0 \%$

$-0.5 \%$

$-3.0 \%$

$-5.5 \%$

$-8.0 \%$ $\begin{array}{llllll}0 & 0.2 & 0.4 & 0.6 & 0.8 & 1.0\end{array}$

(b)

Figure 17: Tracing policy efficiency in an office building and in a school. Growth or decrease rate of the number of newly infected people and efficiency of the policies for the InVS15 dataset (Figure 17a) and the High_School13 dataset (Figure 17b).

The fact that all policies are effective for both dataset for $\varepsilon_{I}=0.8$ is well reflected in the time evolution of the false negatives (Figure 18a and Figure 19a). Indeed, in all cases the number of false negatives peaks at around 5 days and then rapidly decays to zero, as it is expected since 


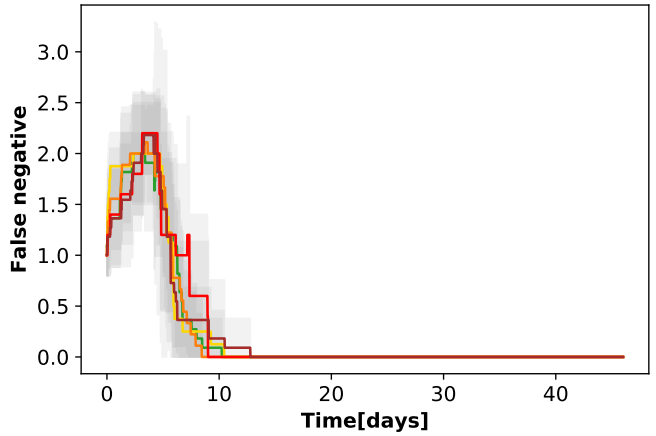

(a)

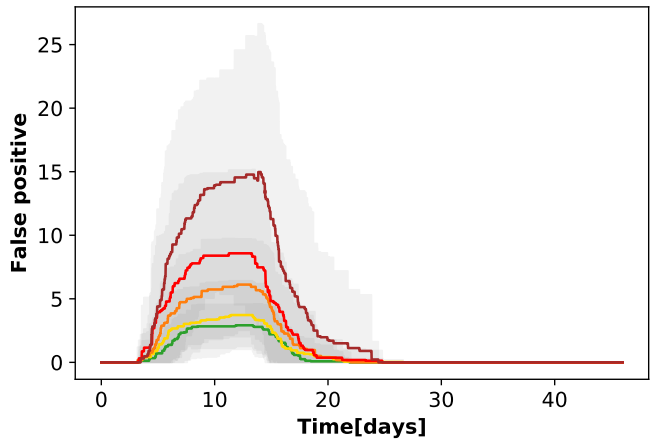

(b)

\begin{tabular}{|c|c|c|}
\hline & $\begin{array}{c}\text { Total } \\
\text { quarantines }\end{array}$ & $\begin{array}{c}\text { Attack } \\
\text { rate }\end{array}$ \\
\hline$\bullet$ & $4 \pm 2$ & $0.16 \pm 0.15$ \\
\hline$\bullet$ & $6 \pm 3$ & $0.088 \pm 0.09$ \\
\hline$\bullet$ & $9 \pm 5$ & $0.048 \pm 0.05$ \\
\hline$\bullet$ & $21 \pm 4$ & $0.050 \pm 0.03$ \\
\hline$\bullet$ & $28 \pm 15$ & $0.044 \pm 0.03$ \\
\hline
\end{tabular}

Figure 18: False positive and negative in quarantines of an office building. Temporal evolution of the numbers of false negatives (Figure 18a) and false positives (Figure 18b) of the InVS15 dataset, for the five different policies and assuming an isolation efficiency of $\varepsilon_{I}=0.8$. The graphs report the mean and standard deviation over 20 independent runs. The table reports mean and standard deviation of the total number of distinct individuals who have been quarantined over the whole simulation timeline and the percentage of those among them who were effectively infected (true positive), corresponding to the attack rate. 


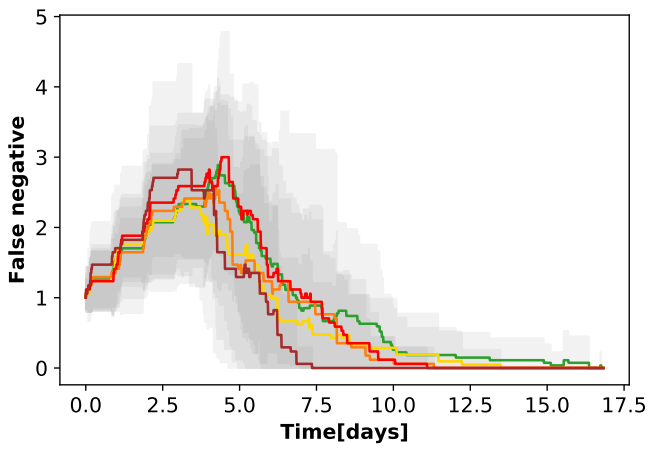

(a)

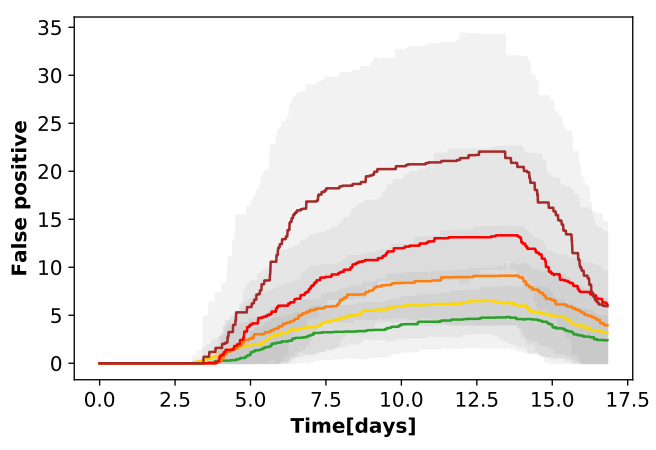

(b)

\begin{tabular}{|c|c|c|}
\hline & $\begin{array}{c}\text { Total } \\
\text { quarantines }\end{array}$ & $\begin{array}{c}\text { Attack } \\
\text { rate }\end{array}$ \\
\hline$\bullet$ & $8 \pm 3$ & $0.21 \pm 0.14$ \\
\hline$\bullet$ & $11 \pm 3$ & $0.16 \pm 0.08$ \\
\hline$\bullet$ & $15 \pm 7$ & $0.14 \pm 0.05$ \\
\hline$\bullet$ & $24 \pm 9$ & $0.08 \pm 0.05$ \\
\hline$\bullet$ & $34 \pm 13$ & $0.062 \pm 0.03$ \\
\hline
\end{tabular}

Figure 19: False positive and negative in quarantines of a school. Temporal evolution of the numbers of false negatives (Figure 19a) and false positives (Figure 19b) of the High_School13 dataset, for the five different policies and assuming an isolation efficiency of $\varepsilon_{I}=0.8$. The graphs report the mean and standard deviation over 20 independent runs. The table reports mean and standard deviation of the total number of distinct individuals who have been quarantined over the whole simulation timeline and the percentage of those among them who were effectively infected (true positive), corresponding to the attack rate. 


\section{Figures}

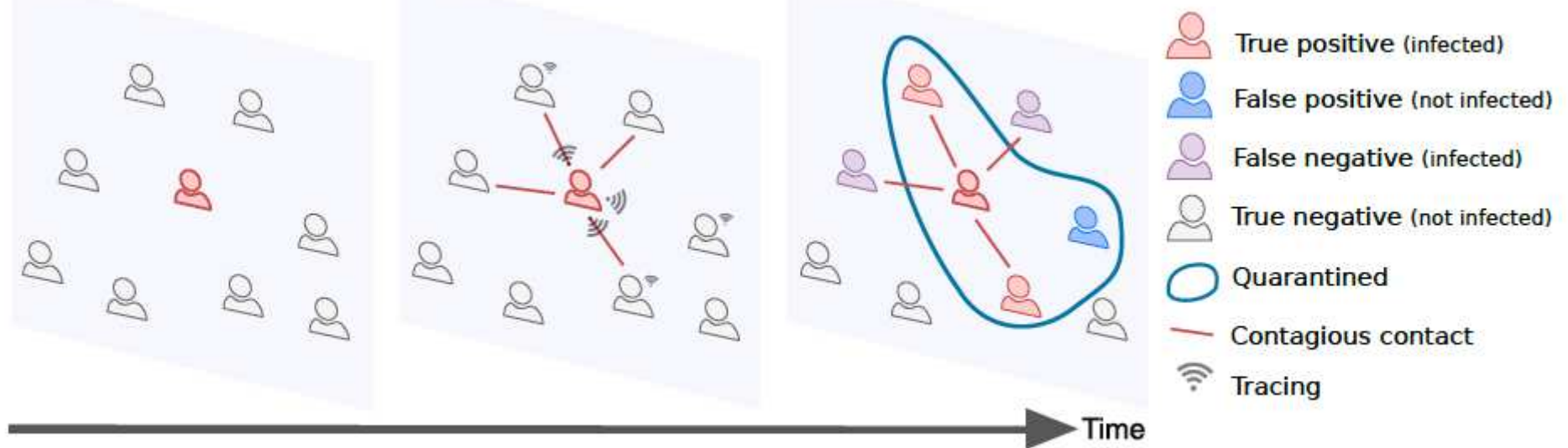

\section{Figure 1}

The contacts among users of the contact tracing app are registered through via the app. As soon as an individual is identified as infected $\mathrm{s} / \mathrm{he}$ is isolated, and the tracing and quarantine policy is implemented. Depending on the policy design, the number of false positives and false negatives may vary significantly.

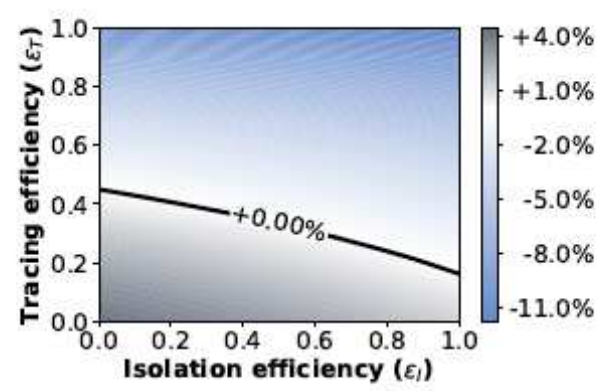

(a)

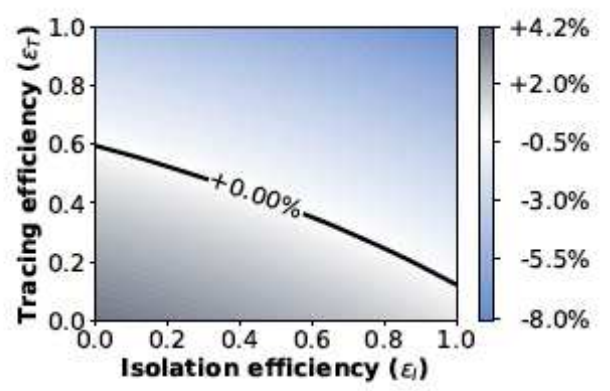

(b)

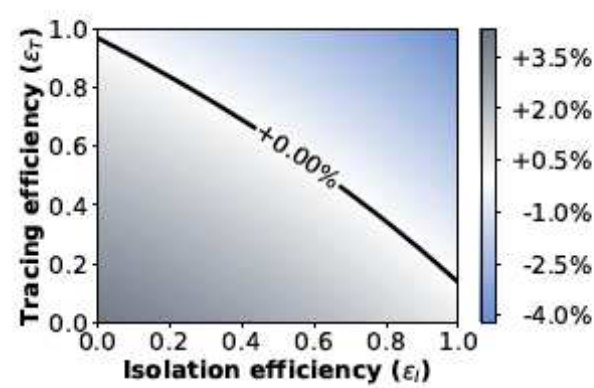

(c)

\section{Figure 2}

Growth or decrease rate of the number of newly infected individuals, assuming either that all the infected people can eventually be identified and isolated (Figure 2a); or that only symptomatic people can be isolated with $20 \%$ of infected individuals asymptomatic (Figure 2 b); or that only symptomatic people can be isolated with $40 \%$ of infected individuals asymptomatic (Figure $2 \mathrm{c}$ ). In all settings the cases are reported with a delay of 2 days. 


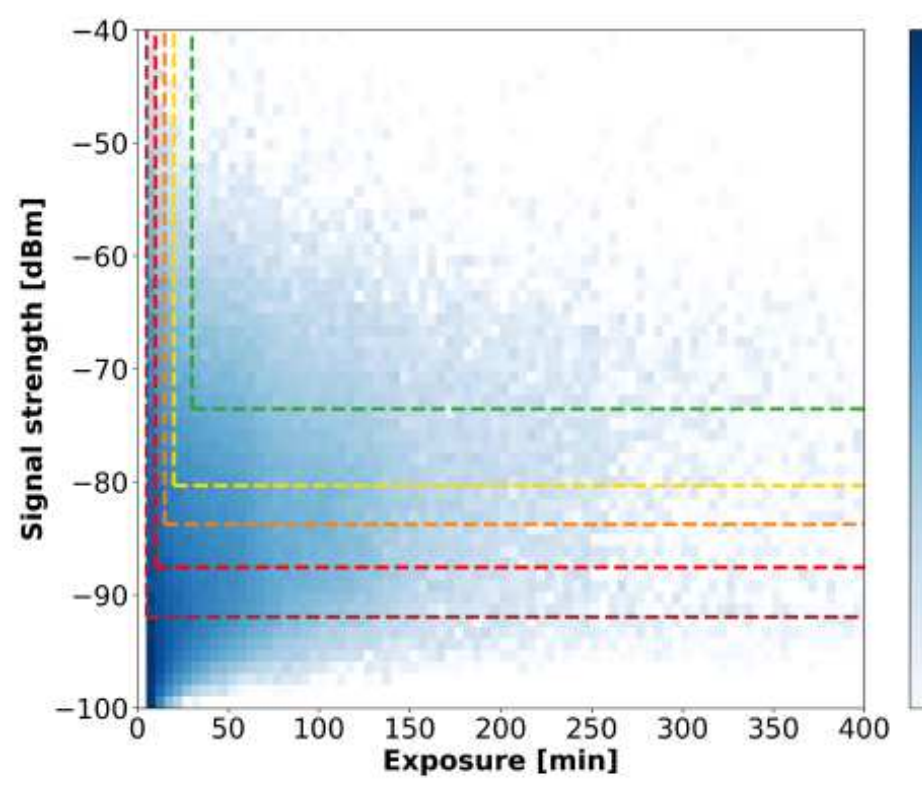

$10^{4}$
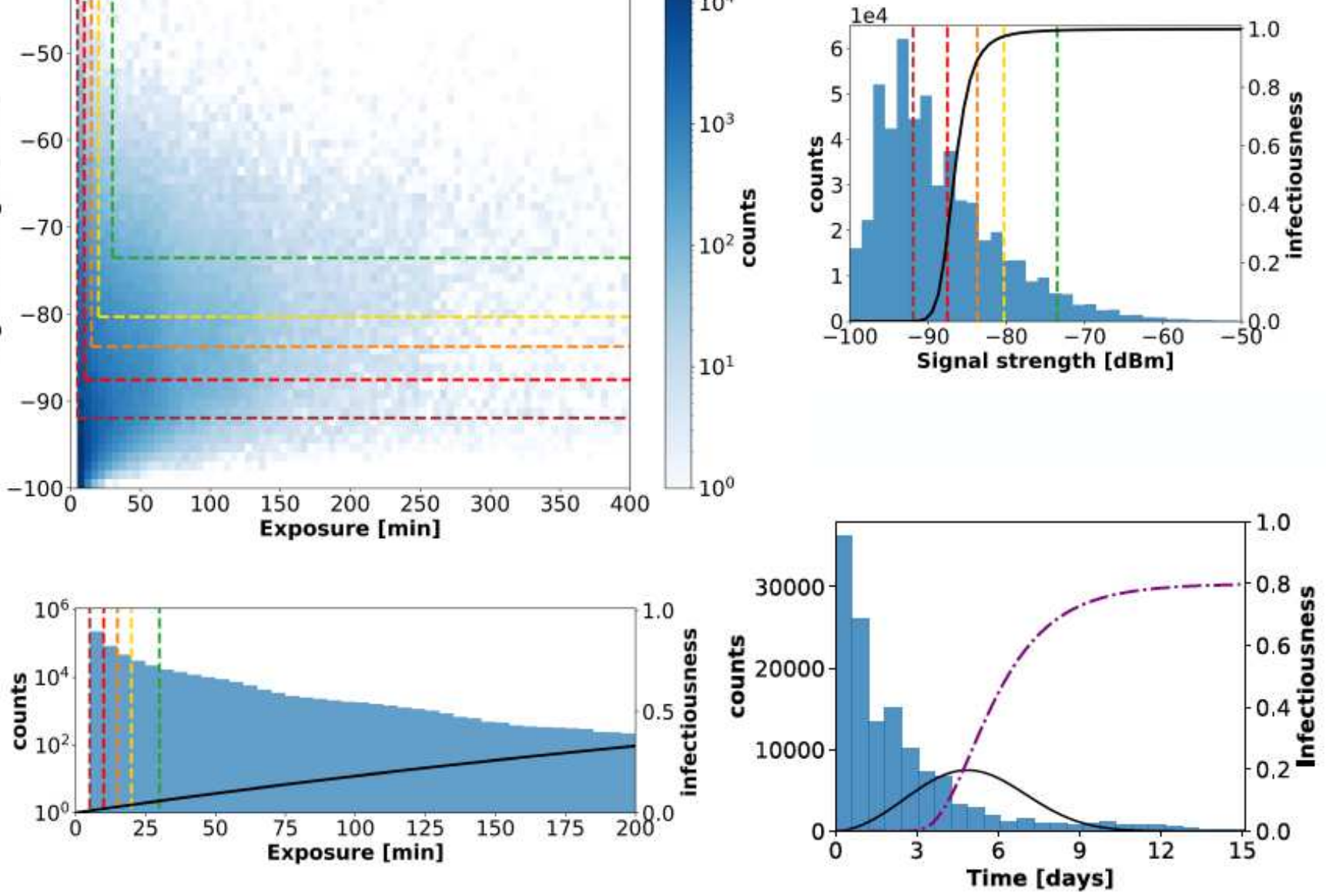

Figure 3

The top left panel shows a scatterplot of signal strength vs duration for all contact events in the CNS dataset, and displays the thresholds defining the various policies: the contacts identified as "at risk" are those included in the areas identified by the colored lines. Top right and bottom left panels separately depict the distributions of signal strength and duration, together with the infectiousness functions wdist and wexposure respectively (black curves), see Table 3 in Supplementary Information for their analytical form. The bottom right panel shows the distribution of time elapsed between the infection of an individual and their successive contacts, obtained with $\varepsilon l=0.8$ and for Policy 5 in the CNS dataset. The black curve shows the normalized infectiousness $\omega(\tau)$ as a function of time, and the purple dashed line is the cumulative probability to detect an infected person $s(\tau)$. 


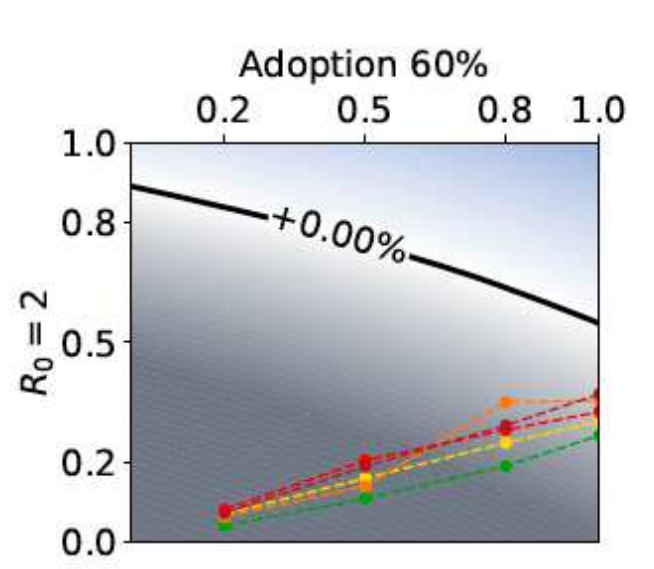

\section{Isolation efficiency $\left(\varepsilon_{l}\right)$}

Adoption $80 \%$
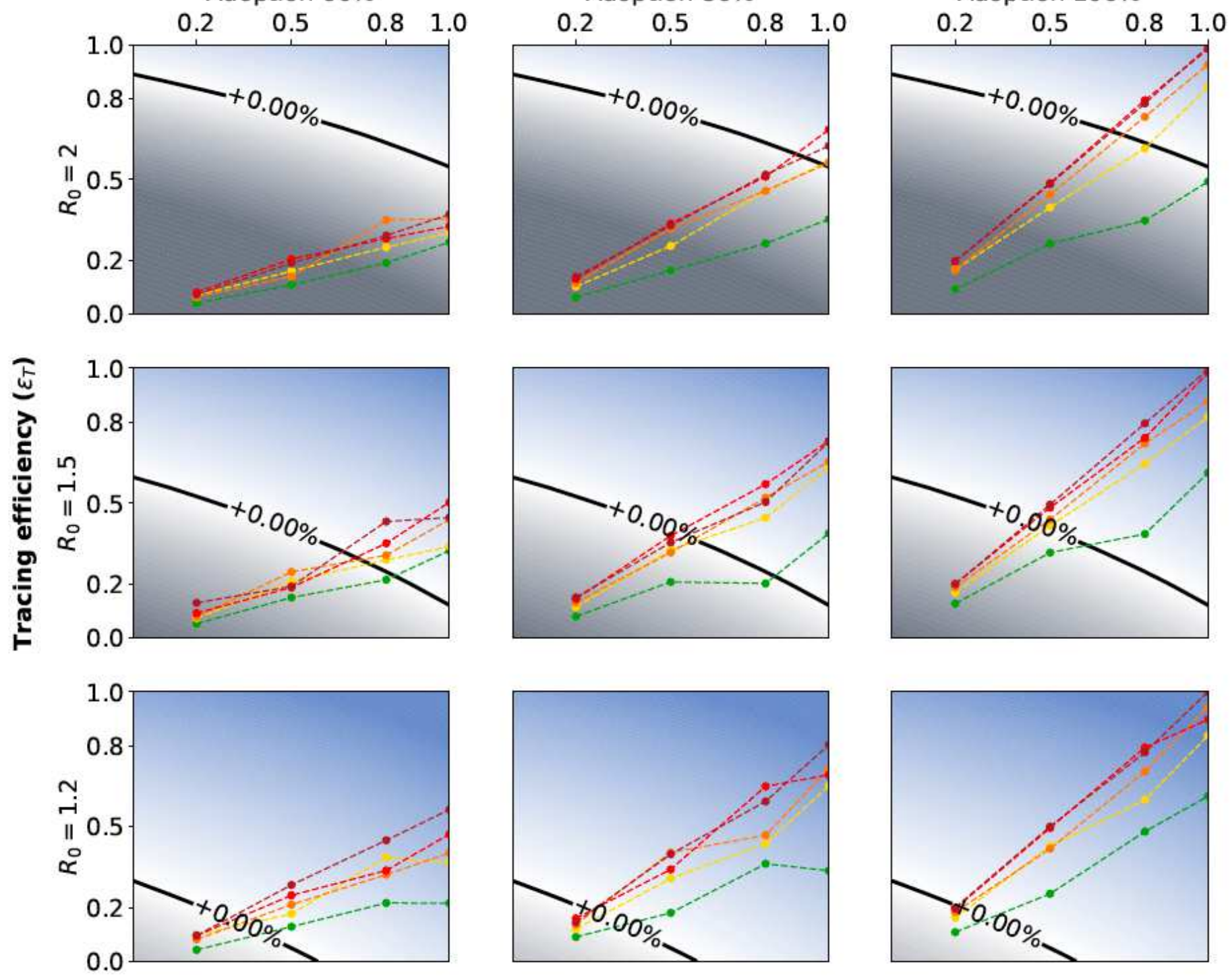

\section{Figure 4}

Tracing policy efficiency. Growth or decrease rate of the number of newly infected people assuming that symptomatic people can be isolated and that an additional $50 \%$ of asymptomatic can be identified via randomized testing. The points correspond to the parameter pairs such that $\varepsilon$ is an input and $\varepsilon T$ an output of the simulations on real contact data, for the five policies. The different scenarios are defined by an app adoption level of $60 \%, 80 \%$, or $100 \%$ (from left to right), and by a value of R0 equal to $2,1.5$, or 1.2 (from top to bottom). 


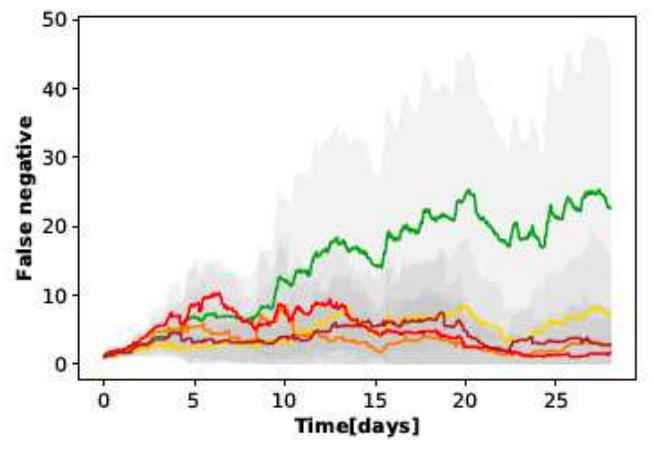

(a)

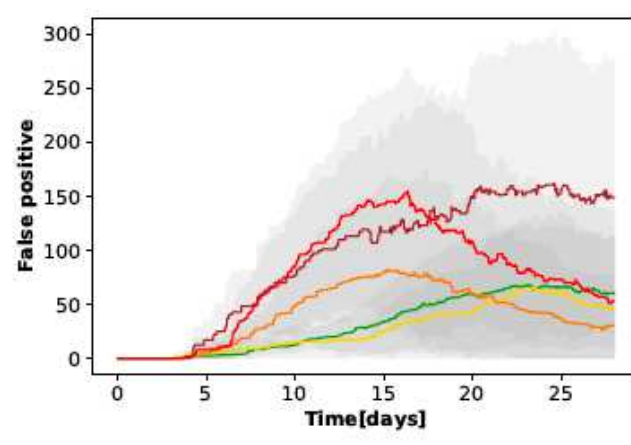

(b)

\begin{tabular}{|c|c|c|}
\hline & $\begin{array}{c}\text { Total } \\
\text { quarantines }\end{array}$ & $\begin{array}{c}\text { Attack } \\
\text { rate }\end{array}$ \\
\hline$\bullet$ & $145 \pm 102$ & $0.16 \pm 0.11$ \\
\hline$\bullet$ & $165 \pm 111$ & $0.097 \pm 0.04$ \\
\hline$\bullet$ & $203 \pm 145$ & $0.088 \pm 0.04$ \\
\hline$\bullet$ & $274 \pm 110$ & $0.061 \pm 0.02$ \\
\hline$\bullet$ & $282 \pm 159$ & $0.036 \pm 0.01$ \\
\hline
\end{tabular}

\section{Figure 5}

False positive and negative in quarantines with $80 \%$ app adoption. Temporal evolution of the numbers of false negatives (Figure 5a) and false positives (Figure 5b) for the five different policies, assuming an isolation efficiency of $\varepsilon l=0.8$, an $80 \%$ app adoption level. The graphs depict the mean and standard deviation over 20 independent runs. The table reports mean and standard deviation of the total number of distinct individuals who have been quarantined over the whole simulation timeline and the percentage of those among them who were effectively infected (true positive), corresponding to the attack rate. 